\title{
Estimated optical constants of gypsum in the regions of weak absorptions: Application of scattering theories and comparisons to independent measurements
}

\author{
Ted L. Roush, ${ }^{1}$ Francesca Esposito, ${ }^{2}$ George R. Rossman, ${ }^{3}$ and Luigi Colangeli ${ }^{2}$ \\ Received 19 March 2007; revised 15 June 2007; accepted 12 July 2007; published 12 October 2007.
}

[1] Diffuse reflectance spectra of multiple grain size fractions are used to estimate the optical constants of gypsum over the $0.4-15 \mu \mathrm{m}$ wavelength region. Two independent scattering theories are used to iteratively determine the imaginary index of refraction from the measured reflectance. We compare the results of these two with each other and with imaginary indices of gypsum reported in the literature. We find that the scattering theory results are more sensitive in the infrared to weak spectral features that are clearly distinguished in the diffuse reflectance spectra. However, we find the scattering results provide a poor determination of the optical constants in the regions of relatively strong absorptions. At visible and near-infrared wavelengths we provide a comparison to the results obtained from analysis of the diffuse reflectance to results obtained from direct transmission measurements of several gypsum crystals having different sample thicknesses. We find the simplest models of the transmission reproduce the observed spectra. The different sample thicknesses for the transmission measurements and different grain sizes in the scattering theories allow evaluation of the imaginary index of refraction over a wide range of values. We find the imaginary index of refraction determined from scattering theories is in remarkable agreement with those obtained from transmission spectra. Different models of the reflectance and transmittance have associated strengths and weaknesses, and we conclude that combining these models provides a more accurate determination of the optical constants of a material when compared to using each separately. We combine the resulting real and imaginary indices of refraction with those reported at infrared wavelengths to provide values covering visual, near-infrared, and infrared wavelengths $\left(0.4-333 \mu \mathrm{m}, 25000-30 \mathrm{~cm}^{-1}\right)$.

Citation: Roush, T. L., F. Esposito, G. R. Rossman, and L. Colangeli (2007), Estimated optical constants of gypsum in the regions of weak absorptions: Application of scattering theories and comparisons to independent measurements, J. Geophys. Res., 112, E10003, doi:10.1029/2007JE002920.

\section{Introduction/Background}

[2] Recent and ongoing remote and in situ observations indicate that sulfates are present in variable abundances in several locations on Mars [e.g., Squyres et al., 2004; Christensen et al., 2004; Klingelhöfer et al., 2004; Rieder et al., 2004; Bibring et al., 2005; Langevin et al., 2005; Gendrin et al., 2005; and references therein]. In addition, the Mars Reconnaissance Orbiter (MRO) is equipped with an imaging spectrometer (MRO-CRISM) that is returning hyperspectral data at higher spatial resolution [Murchie et al., 2003] than the OMEGA instrument on the Mars Express Mission [Bibring et al., 2005]. Thus the optical properties of

\footnotetext{
${ }^{1}$ Planetary Systems Branch, Space Sciences and Astrobiology Division, NASA Ames Research Center, Moffett Field, California, USA.

${ }^{2}$ INAF, Osservatorio Astronomico di Capodimonte, Napoli, Italy.

${ }^{3}$ Division of Geological and Planetary Sciences, California Institute of Technology, Pasadena, California, USA.

Copyright 2007 by the American Geophysical Union. 0148-0227/07/2007JE002920
}

sulfates, in general, are of interest to quantitative interpretation of this increasing volume of remotely sensed data. This is because optical constants describe how a material interacts with electromagnetic radiation and represent the fundamental values used in radiative transfer calculations describing a variety of physical environments. Such environments include atmospheres where aerosols are present, planetary and satellite regoliths, and circumstellar dust clouds.

[3] Here we focus upon gypsum because of its applicability due to its identification on Mars. Also, gypsum is a mineral that is readily available in samples sizes that are suitable for study using a variety of spectral measurements. In the mid-infrared there are several studies reporting the optical constants of gypsum that can be used in evaluating our approach used in the current study. Most importantly, there is a general lack of data regarding the optical constants for gypsum at visible and short-infrared wavelengths $(0.4-5 \mu \mathrm{m})$ that are being observed by the spectrometers on Mars Express $(0.4-5 \mu \mathrm{m})$ and MRO $(0.4-3.95 \mu \mathrm{m})$. 


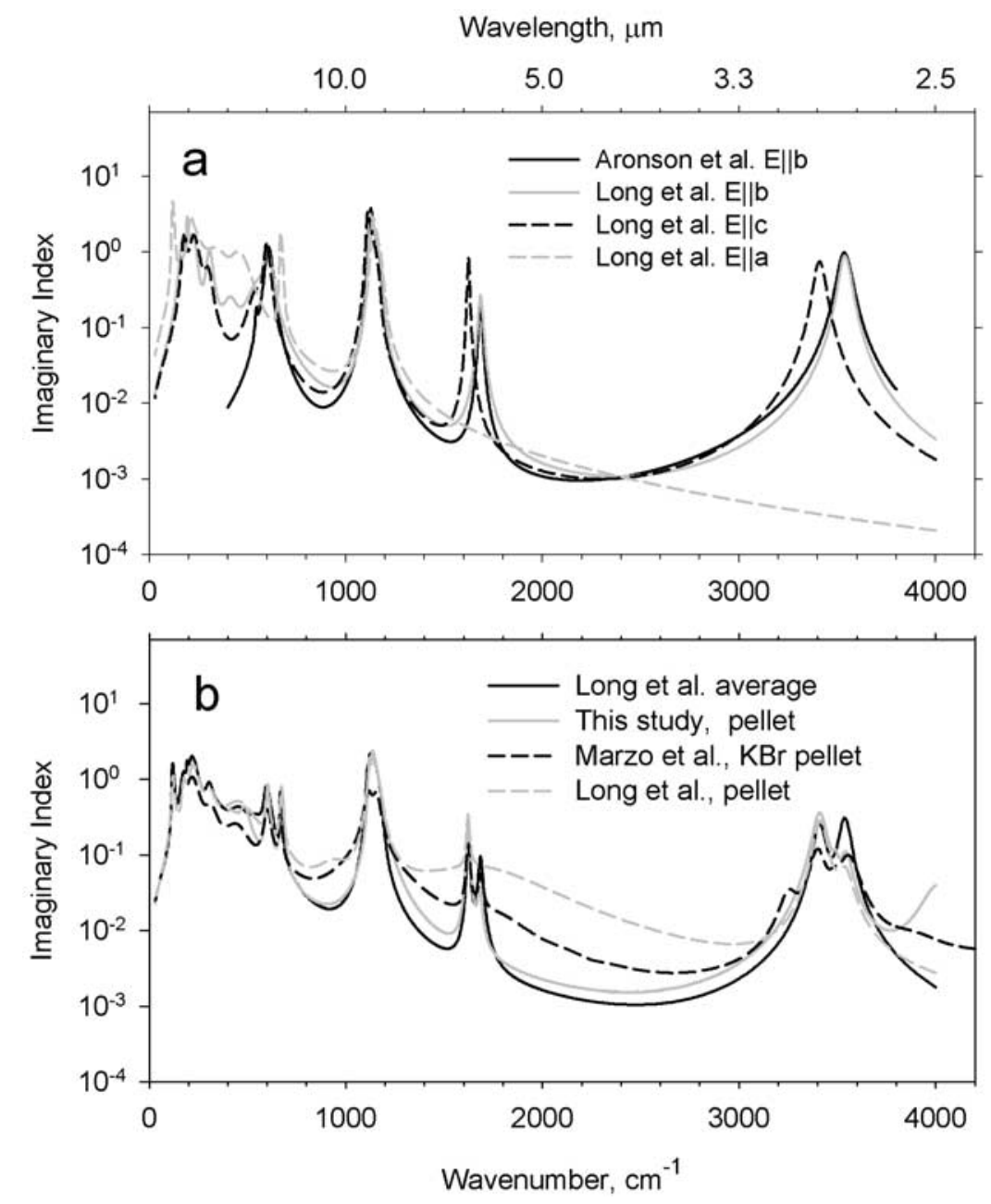

Figure 1. Imaginary index of refraction at infrared wavelengths reported in the literature. (a) Data derived from Fresnel reflectance of oriented crystals (Aronson et al. [1983], solid black line, and Long et al. [1993]). (b) Average of oriented data of Long et al. [1993] (solid black line) compared to values determined from pressed pellets [Long et al., 1993], this study, and the transmission measurement of Marzo et al. [2004].

\subsection{Infrared Determinations of Gypsum Optical Constants}

[4] In the infrared there have been several studies focused at determining the optical constants of gypsum [Aronson et al., 1983; Long et al., 1993; Marzo et al., 2004] and other hydrated sulfates [Roush, 1996]. Aronson et al. [1983] used dispersion analysis to determine the optical constants of gypsum along two different crystallographic planes from the specular reflectance of highly polished surfaces. Using their tabulated oscillator parameters the real $(n)$ and imaginary $(k)$ indices of refraction were calculated and the results are shown in Figure 1a. Long et al. [1993] also used dispersion analysis to determine $n$ and $k$ of gypsum along three different optical axes and a pressed pellet from the specular reflectance of these surfaces. Using their tabulated oscillator parameters, $n$ and $k$ were calculated and the results are also shown in Figures 1a and 1b. As part of the current study (see section 2.2), a compressed pellet of pure material was created and the specular reflection from this pellet was measured. A dispersion analysis was used to determine $n$ and $k$ from these measurements and these are shown in
Figure 1b. Marzo et al. [2004] used dispersion analysis to determine $n$ and $k$ of gypsum from the transmission of fine particles suspended in a $\mathrm{KBr}$ matrix and their resulting values are also shown in Figure 1b. Here we average the results presented by Long et al. [1993] for each axis into a single value because we believe the particulate nature of the samples measured in diffuse reflectance result in a random orientation of the grains. This average $k$ value is also shown in Figure $1 \mathrm{~b}$. This average value exhibits dual peaks in the $500-700,1600-1700$, and $3400-3700 \mathrm{~cm}^{-1}$ region similar to the values determined from reflection and transmission measurements of the pressed pellets. This supports our suggestion that contributions due to each optical axis may be present in randomly oriented samples.

\subsection{Measurements of Gypsum at Shorter Wavelengths, $<5 \mu \mathrm{m}$}

[5] Measurements of gypsum, and other sulfates, at visible, near-infrared, and mid-infrared wavelengths (0.4$5 \mu \mathrm{m})$ have existed for over 100 years. Coblentz [1906] measured the transmission and Fresnel reflectance of rela- 

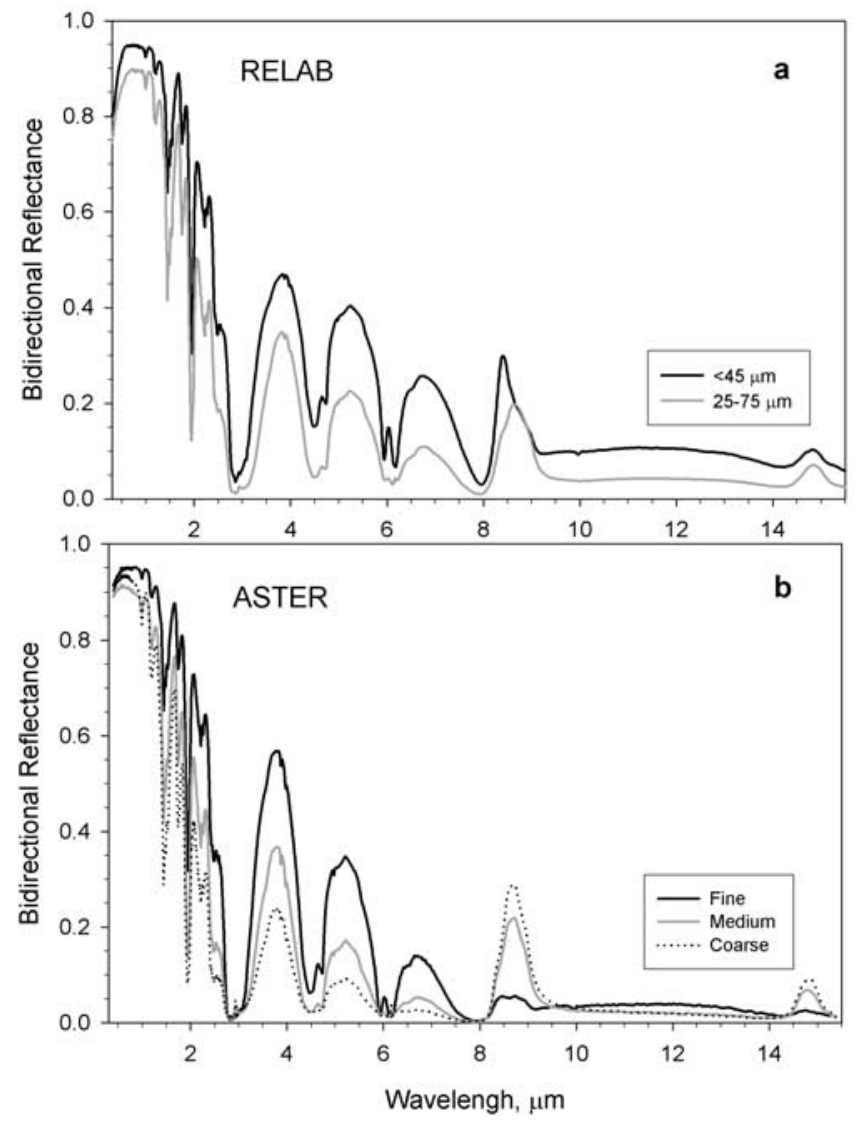

Figure 2. Diffuse reflectance spectra of samples from (a) RELAB and (b) ASTER online spectral libraries.

tively large crystals of various minerals in the $\sim 1-13 \mu \mathrm{m}$ wavelength range $\left(10000-770 \mathrm{~cm}^{-1}\right)$. On the basis of the differences observed between selenite $\left(\mathrm{CaSO}_{4} \bullet 2 \mathrm{H}_{2} \mathrm{O}\right.$, gypsum) and anhydrite $\left(\mathrm{CaSO}_{4}\right)$ and various anhydrous sulfates (e.g., $\mathrm{SrSO}_{4}$ and $\mathrm{PbSO}_{4}$ ), Coblentz [1906] assigned a band observed near $4.55 \mu \mathrm{m}\left(\sim 2195 \mathrm{~cm}^{-1}\right)$ to the sulfate anion and suggested a relationship with another band seen in reflectance at $\sim 8.5-9.1 \mu \mathrm{m}\left(\sim 1175-1100 \mathrm{~cm}^{-1}\right)$.

[6] Hovis [1966] reports the diffuse reflectance spectrum of a particulate sample of gypsum in the $0.4-6 \mu \mathrm{m}$ region. The spectrum exhibits many features due to the molecular water but in addition a notable, broad, strong feature centered near $4.5 \mu \mathrm{m}\left(\sim 2222 \mathrm{~cm}^{-1}\right)$ is present, but is not discussed [Hovis, 1966, Figure 3]. Subsequently, Fink and Burk [1971] measured the diffuse reflectance spectra of particulate samples of reagent materials including several sulfates. All but two sulfate samples exhibited a reflectance minimum between 4 and $5 \mu \mathrm{m}$ that were attributed to a combination mode of the infrared fundamentals of the sulfate anion [Fink and Burk, 1971]. Salisbury et al. [1991] present the spectra of anhydrite and gypsum and attribute a feature near $4.6 \mu \mathrm{m}\left(\sim 2174 \mathrm{~cm}^{-1}\right)$, seen in both spectra, to a combination mode of the sulfate anion. Blaney and McCord [1995] report the diffuse reflectance spectra of three sulfates, including anhydrite. All spectra exhibited a minimum near $4.5 \mu \mathrm{m}$ that was attributed to the first overtone of the $\nu_{3}$ fundamental associated with the $\mathrm{SO}_{4}$ anion [Blaney and McCord, 1995]. Brown University operates a Reflectance Experimental Laboratory (RELAB) to study the reflectance properties of materials [Pieters and Hiroi, 2004]. As part of the operation an online spectral library (http://lf314-rlds.geo.brown.edu/) is maintained that allows searching and retrieval of archived data. A search of this library yields numerous sulfates, including gypsum. As shown in Figure 2a, the spectra of the gypsum samples from the RELAB data archive exhibit the reflectance minimum near $4.5-4.6 \mu \mathrm{m}\left(\sim 2175-2225 \mathrm{~cm}^{-1}\right)$, seen by previous researchers. As part of operations associated with the Advanced Spaceborne Thermal Emission and Reflection Radiometer (ASTER) instrument in orbit around the Earth, the ASTER group maintains an online spectral library (http://speclib.jpl.nasa.gov/). A search of this library yields numerous sulfates, including gypsum. As shown in Figure $2 b$, the spectra of the gypsum samples from the ASTER data archive exhibit the reflectance minimum near $4.5-4.6 \mu \mathrm{m}$, seen by previous researchers. Thus there is ample independent evidence of spectral features near 4.5$4.6 \mu \mathrm{m}$ associated with sulfates. Yet, as illustrated in Figure 1, few of the determinations of optical constants of gypsum suggest the presence of such a feature. This provides additional motivation for determining the optical constants of gypsum in the visible, near-infrared, and midinfrared wavelength region.

[7] In the following sections we describe the laboratory data used in our analyses. We then describe our various analytical approaches to deriving $n$ and $k$ for gypsum using diffuse reflectance and transmission measurements at visible, near-infrared, and mid-infrared wavelengths. This is followed by a discussion and comparison of the results from the different analytical methods. We then place our results into context with results of optical constants determined for gypsum at infrared wavelengths and then compile a final recommendation of the optical constants for gypsum.

\section{Laboratory Data}

\subsection{Diffuse Reflectance Data}

[8] The RELAB online spectral library contains both spectral data and information concerning sample acquisition, characterization, preparation, and spectral measurements. The gypsum samples included in this study are $<45$ micron (SF-EAC-041-A/LASF41A) and 2575 micron (CC-JFM-016-B/F1CC16B). These samples have been chosen because they are sieved to produce a limited size range and the spectral data are available over a wide spectral range: $0.3-26 \mu \mathrm{m}$. Two spectrometers were used to acquire the reflectance spectra. The first measures the bidirectional reflectance with an incident angle of $30^{\circ}$ and reflectance angle of $0^{\circ}$ and covers the 0.3-2.6 $\mu \mathrm{m}$ region. The second measures the biconical reflectance and covers the $0.8-26 \mu \mathrm{m}$ region. Typically the biconical reflectance data are scaled to agree with the bidirectional data and then combined in the region of wavelength overlap. Here we use data from the site that was already combined and these are shown in Figure 2a.

[9] The ASTER online spectral library contains both spectral data and information concerning sample acquisition, characterization, preparation, and spectral measurements. The gypsum sample included in this study (SO-02B) was separated into three sieve size fractions of $<45 \mu \mathrm{m}$, 45-125 $\mu \mathrm{m}$, and $125-500 \mu \mathrm{m}$. Two spectrometers were 
Table 1. Elemental Abundances of Selenite Sample Used for Fresnel Measurements

\begin{tabular}{ccc}
\hline Element & Concentration, wt. \% & Oxide Abundance \\
\hline $\mathrm{S}$ & $17.4 \pm 0.6$ & $\mathrm{SO}_{3} 43.4 \pm 1.6 \%$ \\
$\mathrm{Ca}$ & $33.8 \pm 0.7$ & $\mathrm{CaO} 47.2 \pm 0.1 \%$ \\
$\mathrm{O}$ & $39.5 \pm 0.8$ & \\
\hline
\end{tabular}

used to acquire the reflectance spectra. Both collected directional-hemispherical reflectance with one covering the $0.4-2.5 \mu \mathrm{m}$ region and the other the $2.0-15 \mu \mathrm{m}$ region. These individual data sets were combined in the region of overlap. The bidirectional reflectance was calculated from equation (4) of Shkuratov and Grynko [2005]. Specifically, $\log \mathrm{R}_{\mathrm{b}}\left(30^{\circ}\right)=1.088 \log \mathrm{R}_{\mathrm{h}}$, where $\mathrm{R}_{\mathrm{b}}$ is the bidirectional reflectance with incidence and emission angles of $30^{\circ}$ and $0^{\circ}$, respectively, and $\mathrm{R}_{\mathrm{h}}$ is the hemispherical reflectance. The resulting values are shown in Figure $2 b$ for each grain size. Examination of the spectra in Figure $2 b$ shows that the reflectance of the coarse-grained sample is greater than the medium grained sample at wavelengths $<1 \mu \mathrm{m}$. This spectral behavior is suspicious since as the grain size increases, the path length of photons through the material should increase and the effect should be increasing absorption resulting in a decrease in reflectance. As a result, we do not use the coarse-grained data in any further analyses.

\subsection{Mid-Infrared Fresnel Measurements}

[10] Specular reflectance measurements have been performed on a sample of pure selenite in order to compute the optical constants in the MIR via Fresnel's law and Lorentz oscillator model (for the interested reader, more details are given by Roush et al. [1991] and Esposito et al. [2000] and references therein).

[11] Selenite is the colorless and transparent variety of gypsum and it was originally purchased from WARD'S (USA) and provided by Giuseppe Marzo (University of Lecce, Italy). Its elemental composition has been characterized by an energy dispersive X-ray analysis (EDX) performed with a microanalysis system interfaced to a Scanning Electron Microscope (Cambridge Model Stereoscan 360FE) and it is reported in Table 1.

[12] The sample was ground and sieved to $<20 \mu \mathrm{m}$. Then it was ground again in an agate mortar for about 5-10 minutes. Using this procedure a particle size in the submicrometer range was generally obtained, as verified via scanning-electron microscopy. This very fine powder was subsequently pressed between two optically worked stainless steel surfaces. Four pellets with high reflective surfaces were obtained. The total (diffuse plus specular) and diffuse bi-conical reflectance of the pellets were acquired using a Fourier transform Michelson interferometer (Bruker Equinox 55) equipped with a diffuse/ specular accessory (Graseby Specac Model Selector). The specular reflectance at $45^{\circ}$ of our samples was obtained by calculating the difference between total and diffuse reflectance spectra. All the measurements were performed in a dry air environment in order to reduce the content of atmospheric $\mathrm{CO}_{2}$ and $\mathrm{H}_{2} \mathrm{O}$ in the sample chamber. A quartz pellet with a gold coating was used as reference in the measurements. For each pellet a set of $10-15$ measurements were acquired in order to minimize the effect of possible environmental variations. A spectrum of the reference was acquired for each three measure- ments of the sample in order to compensate for any instrumental effect. To obtain the final absolute specular reflectance the selenite spectra were normalized to the reference and then multiplied by 0.995 , which is the reflectivity of gold in the MIR. The resulting data are shown in Figure 3.

\subsection{Transmission Measurements}

[13] Four samples, from three locations, were used to obtain transmission measurements of gypsum. The thinnest sample, $0.034 \mathrm{~cm}$, is from an unspecified locality in Nevada. The sample from Brookville, in Saline County Kansas, had two thicknesses, 0.1171 and $0.2856 \mathrm{~cm}$, respectively. The sample from Wayne County, Utah is $1.4293 \mathrm{~cm}$ thick. This range of sample thickness allows us to address the significant absorbance of gypsum that occurs over the visible and short wavelength infrared range. Each sample exhibited the characteristic tabular shape associated with the perfect cleavage of gypsum along the $\{010\}$ plane. Without additional preparation each sample was placed in the spectrometer with the $\{010\}$ plane perpendicular to the incident beam. Two transmission measurements were acquired for each sample with the incident beam polarized parallel and perpendicular to the $\{010\}$ plane resulting in measurements approximately parallel to the $\mathrm{Z}$ and $\mathrm{X}$ crystallographic axes, respectively. Crystallographic axes are commonly referred to as $\mathrm{X}, \mathrm{Y}$, and $Z$, either lower or upper case. Distances to the crystallographic unit cell edge along these axes are referred to as a, $\mathrm{b}$, and $\mathrm{c}$, respectively. In the literature $\mathrm{a}, \mathrm{b}$, and $\mathrm{c}$ are commonly used interchangeably with $\mathrm{X}, \mathrm{Y}$, and Z. For comparison to historical data we use the $\mathrm{X}, \mathrm{Y}$, and $\mathrm{Z}$ nomenclature. These crystallographic axes do not necessarily coincide with the optic axes that are typically labeled $\alpha, \beta$, and $\gamma$. These same symbols are commonly used to represent the range of refractive indices along the optic axes. Below we relate measurements along $\mathrm{X}$ and $\mathrm{Z}$ to $\alpha$ and $\gamma$ when we illustrate our results for the transmission measurements.

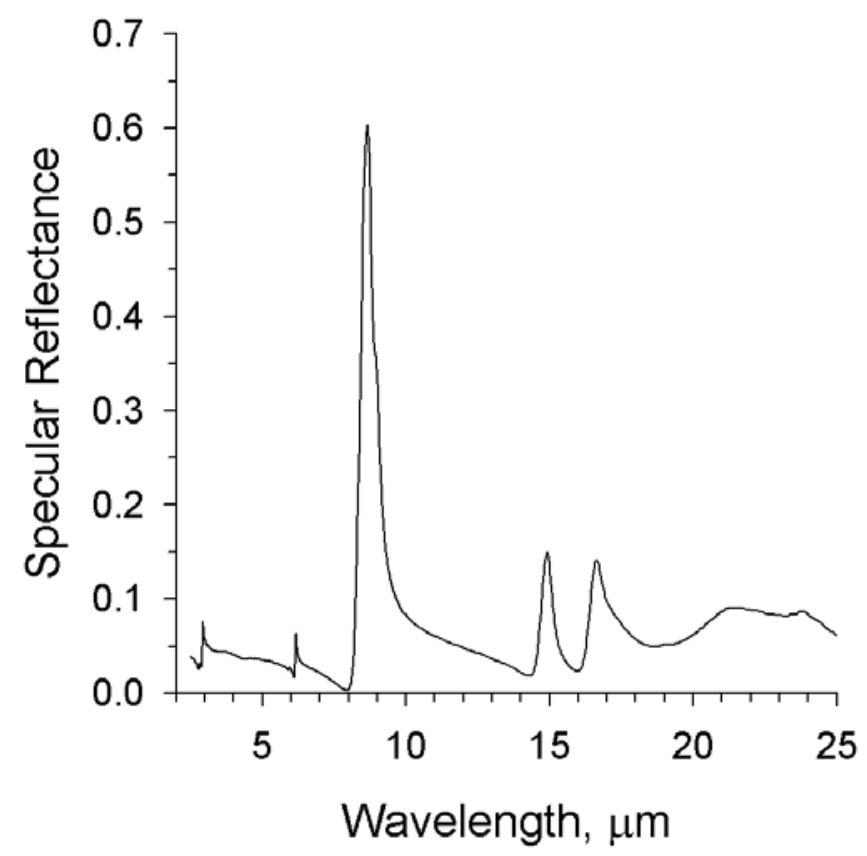

Figure 3. Measured laboratory specular reflectance of gypsum in the IR. 

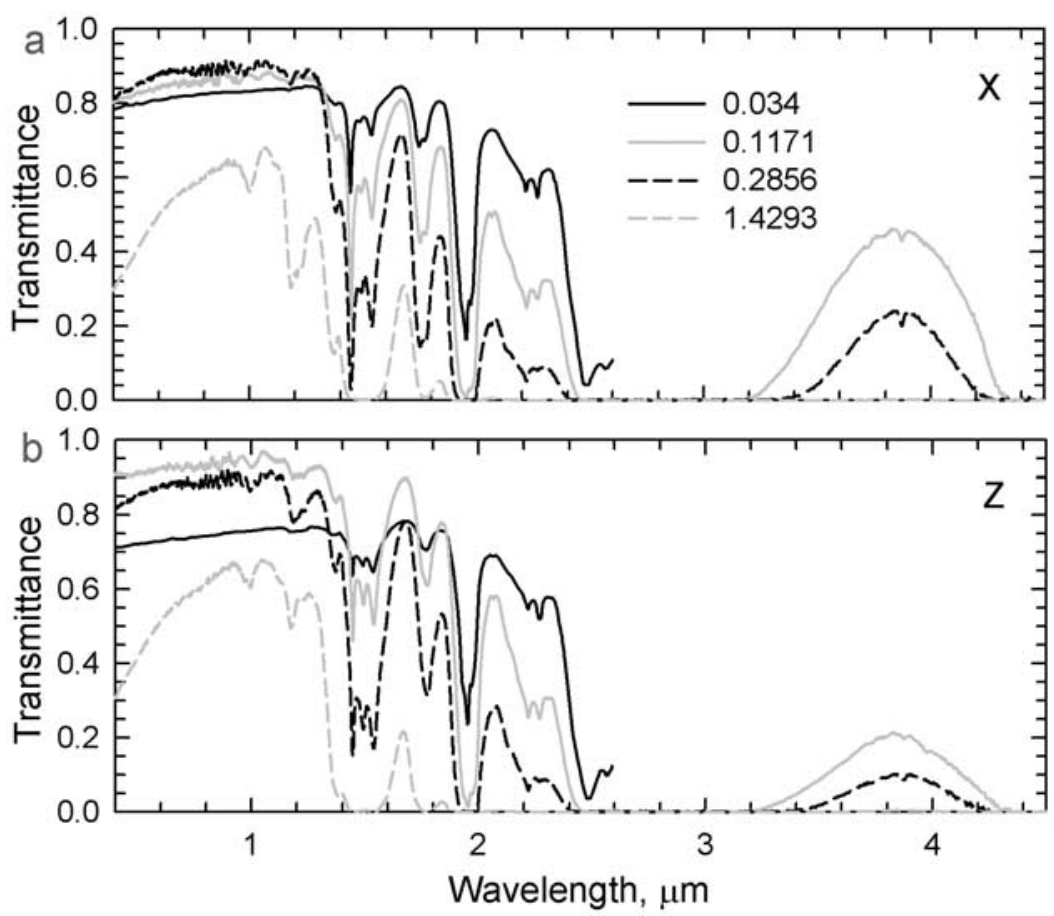

Figure 4. Transmission spectra of gypsum crystals with the incident spectrometer beam polarized (a) perpendicular $(\mathrm{X})$ and (b) parallel $(\mathrm{Z})$ to the $\{010\}$ crystal face. This means the transmitted beam is chiefly oriented along the $\alpha$ and $\gamma$ optical axes, respectively. Line types for each sample thickness, given in $\mathrm{cm}$, are indicated at the top.

[14] The transmissions of the samples are shown in Figure 4. It is clear that a range of sample thicknesses are required to assess the absorption in some of the stronger bands. There is also an indication of scattering in the transmission of the thinnest sample. This is particularly evident in the visible and near-infrared region, $\sim 0.4-1.2 \mu \mathrm{m}$, where the thinnest sample has a noticeably lower transmittance than the next two thicker samples. We return to this issue in derivation of the imaginary index of refraction that is discussed in more detail in section 4.2.

[15] Infrared and near-infrared spectra were obtained with a Thermo-Nicolet Magna 860 FTIR using $\mathrm{KBr}$ and $\mathrm{CaF}_{2}$ beamsplitters, respectively with a room temperature deuterated triglycine sulphate detector. Visible spectra were obtained with a home-built diode array spectrometermicrospectrometer system consisting of two diode-array detectors (1024 channel $\mathrm{Si}$ and 256 channel InGaAs) coupled to a grating spectrometer system via fiber optics to a highly modified NicPlan infrared microscope containing a calcite polarizer. Pairs of conventional $40 \times$ and $10 \times$ objectives were used as objectives and condensers. Spectra were taken after the samples were placed on a rotating state and adjusted for optical extinction under crossed polarizers. A second spectrum was taken after rotating the sample 90 degrees to the second extinction position.

\section{Analytical Approaches for Determining $\boldsymbol{n}$ and $\boldsymbol{k}$ 3.1. Determination From Diffuse Reflectance via Scattering Theories}

[16] Hapke and Wells [1981] and Clark and Roush [1984] describe an approach to determination of the absorption coefficient, $\alpha$, from measured reflectance spectra that relies upon Hapke's description of radiative transfer within particulate surfaces [see Hapke, 1981, 1986, 1993, and references therein]. Since $\alpha$ is related to $k$ via the dispersion relation, $\alpha=4 \pi k / \lambda$, this approach provides a mechanism of determining $k$. More recently, Poulet and Erard [2004] describe an approach for deriving $k$ using Shukhartov's description of radiative transfer within particulate surfaces [Shkuratov et al., 1999].

\subsubsection{Approach Using Hapke's Model}

[17] Roush et al. [1990] and Lucey [1998] used the Hapke approach for determination of $k$ for serpentine, and olivines and pyroxenes, respectively. A brief summary is provided here and the interested reader is referred to Roush et al. [1990] and Lucey [1998] for more details. Beginning with the reflectance spectrum, assume $n$ is constant, and iteratively change $k$ to force the calculated and measured reflectance to agree with one another. Additional leverage is provided in determining $k$ if several grain sizes of the same material are used, with the assumption that the composition is not a function of grain size. Roush [2003, 2005] describes an additional iterative approach that allows determination of $n$ as a function of wavelength using a subtractive Kramers-Kronig analysis as is discussed in more detail in section 3.4.

[18] The various assumptions contained within the Hapke formalism [Hapke, 1993, and references therein] used here are discussed in equations (1)-(6) of Roush [1994] and equations (1)-(4) of Cruikshank et al. [1997]. Here scattering is assumed isotropic, $\mathrm{h}$, the width of the opposition surge, is assumed to be 0.05 , and Hapke's internal scattering parameter, $s$, is set at $10^{-17}$. The first two of these parameters require observations at multiple viewing geometries that were not obtained. The third parameter is poorly 
characterized for natural materials and essentially setting it to zero effectively forces the absorption coefficient to account for all the spectral behavior. The assumption regarding $s$ is investigated and discussed in section 4.1.1.

\subsubsection{Approach Using Shkuratov's Model}

[19] The Shkuratov model [Shkuratov et al., 1999] is a one-dimensional geometrical optics model for spectral reflectance of powdered surfaces. These surfaces are considered composed of semi-transparent particles of arbitrary form, whose sizes $d$ are much greater than the wavelength, $\lambda$, of the incident radiation. Each particle in the model represents an elementary volume of the particulate medium. Multiple reflections within a particle are considered as multiple scattering in a one-dimensional medium (plate) with reflection coefficients obtained from averaging the usual Fresnel coefficients over the incident angle. So, all angular dependencies of reflectance are ignored and the scattering in a system of particles is approximated by the scattering in an equivalent system of plates. The parameters of the model are the optical constants $n$ and $k$, the average path length $S$ in the particle between two internal reflections, and the porosity q. Shkuratov et al. [1999] showed that reflectivities are only very weakly dependent on $q$. The parameter $S$, in the case of transparent particles, should be equal approximately to the average diameter of the particles.

[20] One of the strengths of this model, besides its relative simplicity, is that it is analytically invertible; so it is possible to compute the imaginary part $k(\lambda)$ of the index of refraction from reflectance spectra if estimates for the real part $n$ and the particle size of the sample are available. So, starting from laboratory spectra of characterized samples and assuming $n$ is constant, we are able to compute $k$ starting from equation (13) of Shkuratov et al. [1999].

[21] As in the case of the application of the Hapke's model, we decided to apply the model to spectra of several particle sizes, adjusting the value of each average size in order to minimize the deviations among the values of $k(\lambda)$ obtained. Again, we used the subtractive Kramers-Kronig analysis to compute $n$ from $k$.

\subsection{Determination of $\boldsymbol{n}$ and $\boldsymbol{k}$ via Dispersion Analysis}

[22] Many previous studies describe the application of dispersion analysis to derive $n$ and $k$ of a material in terms of the summation due to a number of classical Lorentz oscillators [see Roush et al., 1991; Roush, 1996; Esposito et al., 2000; and references therein]. Each oscillator is defined by its strength $\left(\rho_{i}\right)$, width $\left(\Gamma_{i}\right)$, and central wavelength $\left(\lambda_{i}\right)$. The other parameter required is the high-frequency dielectric constant $\left(\varepsilon_{\infty}\right)$ that is estimated from values of the real index of refraction, $\varepsilon_{\infty}=\mathrm{n}_{\text {vis }}^{2}$, measured in the visible.

[23] In the analysis it is required that (1) $\rho_{i}, \Gamma_{i}$, and $\lambda_{i}$ are all greater than zero; (2) $\lambda_{i}$ falls within the range of laboratory observations; and (3) the difference between the observed and computed reflectance (i.e., $\chi^{2}$ ) is minimized. Requirement (2) means that the analysis is not sensitive to spectral features that lie outside the wavelength region measured. In the fitting procedure, the initial number of oscillators is chosen on the basis of the number of obvious peaks in the reflectance spectrum; additional oscillators are added to provide an adequate description of the reflectance spectrum if necessary. Given an initial estimate of the oscillator parameters, the reflectivity was calculated and compared to the laboratory measurement. This process was reiterated, using nonlinear least squares techniques [e.g., Bevington, 1969], until optimal values of $n$ and $k$ were determined and $\chi^{2}$ was minimized.

[24] Toon et al. [1976] provide a discussion of the relative errors associated with derivation of $n$ and $k$ using dispersion analysis. They conclude that $\Delta n / n$ is a few percent due to errors associated with the measured reflectivity. In respect to $k$, if $0.01<k<1$, i.e., in the strong bands, then comparison of values derived from dispersion analysis and KramersKronig analysis agree to within a few percent.

\subsection{Determination of $\boldsymbol{k}$ From Transmission Measurements}

[25] The laboratory transmission is the ratio of the detector response with the sample in the spectrometer beam to the detector response without the sample in the spectrometer beam. This implies reflection losses from the air-sample interfaces on both sides of the crystal. These losses remove energy from the transmitted beam relative to when the sample is absent. This "missing" energy could be incorrectly interpreted as absorption. Additionally, because of these reflections at the interfaces, multiple path lengths of light in the beam contribute to the measured transmission. The light transmitted by a slab of material is discussed in detail by Bohren and Huffman [1983] and Heavens [1970]. Full treatment of the transmission of a slab $\left(\mathrm{T}_{\text {slab }}\right)$ is given by equation (2.74) of Bohren and Huffman [1983]:

$$
T_{\text {slab }}=\frac{(1-R)^{2}+4 R \sin ^{2} \psi}{R^{2} e^{-\alpha d}+e^{\alpha d}-2 R \cos (\varsigma+2 \psi)}
$$

where

$$
R=|\tilde{r}|^{2}=\left|\frac{m_{0}-m_{1}}{m_{0}+m_{1}}\right|^{2}, \quad m=n+i k
$$

and

$\psi=\tan ^{-1}\left(\frac{2 n_{0} k_{1}}{n_{1}^{2}+k_{1}^{2}-n_{0}^{2}}\right), \quad 0 \leq \psi \leq \pi, \varsigma=\frac{4 \pi n_{1} d}{\lambda}, \quad \alpha=\frac{4 \pi k_{1}}{\lambda}$

where $d$ is the thickness of the sample, $m_{0}$ is the complex index of refraction of air, $m_{1}$ is the complex index of refraction of the slab material, and $\widetilde{r}$ is the Fresnel reflection coefficient. Transmission measurements are valid only if a measurable amount of light is transmitted by the sample. This requires that $\mathrm{k}_{1}$ is small, with the resulting effect that the $\psi$ term is eliminated. This leads to the modified version of the equation given by equation (2.75) of Bohren and Huffman [1983]:

$$
T_{\text {slab }}=\frac{(1-R)^{2}}{R^{2} e^{-\alpha d}+e^{\alpha d}-2 R \cos \varsigma}
$$

The oscillatory term, $\zeta$, is due to the constructive and destructive interference caused by multiple path lengths through the crystal and can give rise to interference fringes in the transmission spectrum. Bohren and Huffman [1983] 


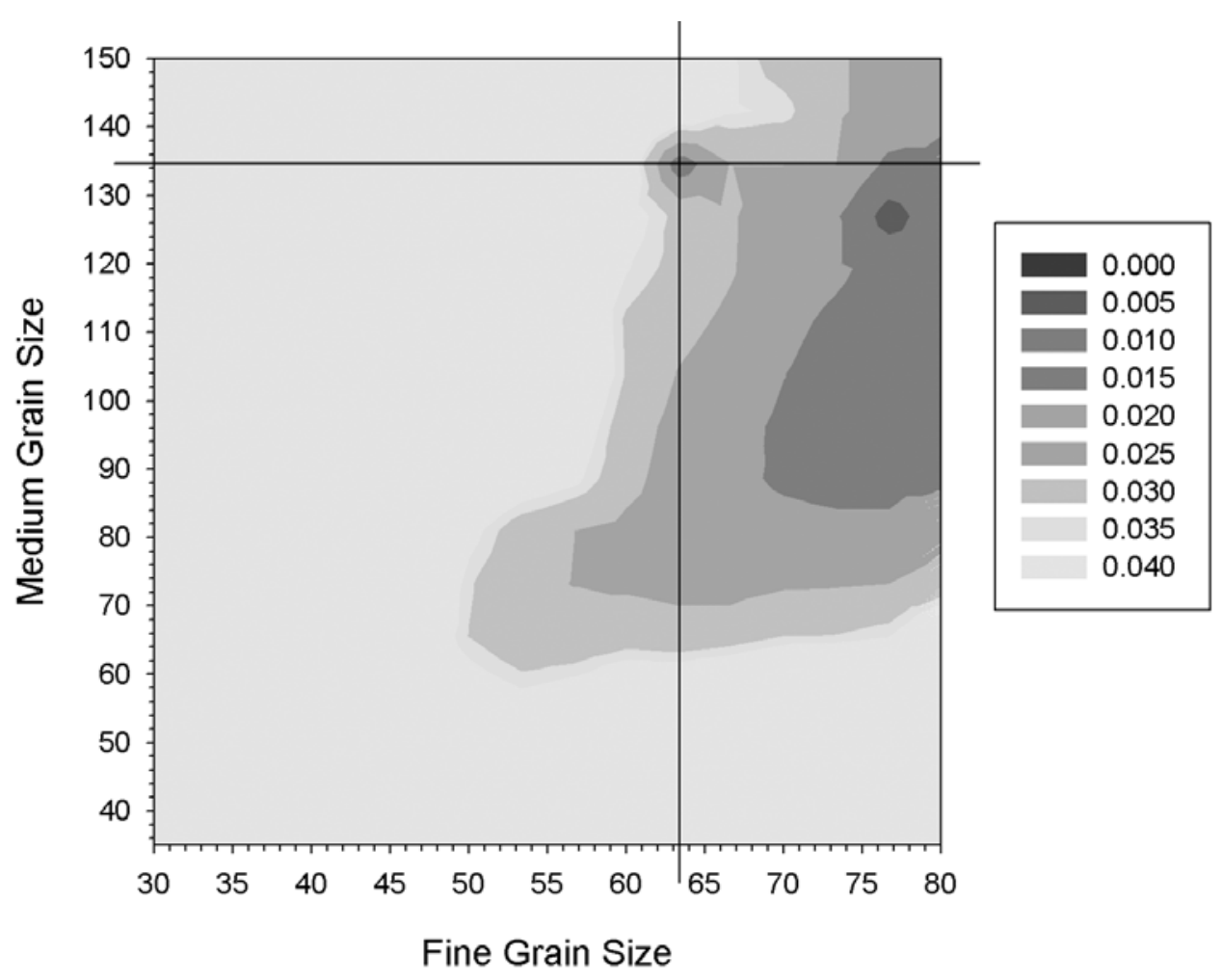

Figure 5. Shaded contour plot showing the resulting $\chi^{2}$ values associated with different diameters $(\mu \mathrm{m})$ used in the Hapke models assuming $s=10^{-17}$. The intersection of the two lines indicates the diameters associated with the lowest $\chi^{2}$ values.

discuss several factors influencing $\zeta$ and that may eliminate these interference fringes and present several equations to estimate the physical requirements: (1) the incident beam is not perfectly monochromatic; (2) the absorption in the slab becomes sufficiently large; (3) the slab surfaces are not perfectly parallel (for the wavelengths considered here the average departure from parallelism $\delta$ must be $\sim 0.05 \mu \mathrm{m}<$ $\delta<\sim 0.4 \mu \mathrm{m}$ ); and (4) the slab surface roughness, $v$, is not negligible $(\sim 0.04 \mu \mathrm{m}<v<\sim 0.31 \mu \mathrm{m})$. If any of these conditions exist, then $\zeta$ is negligible, and a further simplification can be made yielding equation (2.76) of Bohren and Huffman [1983]:

$$
T_{\text {slab }}=\frac{(1-R)^{2} e^{-\alpha d}}{1-R^{2} e^{-2 \alpha d}}
$$

We investigate each of these three models as describing the measured transmission in the analysis discussion below.

\subsection{Determination of $\boldsymbol{n}$ Using Subtractive Kramers-Kronig Analysis}

[26] A subtractive Kramers-Kronig analysis (SK-K) derives $n$ from existing values of $k$ [e.g., Warren, 1984, and references therein] and thus $n$ becomes a function of wavelength. Formal Kramers-Kronig analysis requires data at all wavelengths which is typically unavailable for most studies. However, this problem is addressed by the SK-K approach by incorporating as broad a wavelength domain as is available. In the SK-K analysis the $k$ values are the input parameters, and in addition $\varepsilon_{\infty}$, at a particular wavelength must be specified and is estimated via $\varepsilon_{\infty}=\mathrm{n}_{\mathrm{vis}}^{2}$ [Roush et al., 1991; Roush, 1996]. Here we use 1.524 at $0.5893 \mu \mathrm{m}$. This value corresponds to an average of the values cited for gypsum by Long et al. [1993]. To provide as broad a wavelength coverage as is possible, the $k$ values determined from the various laboratory measurements are ultimately combined with the $k$ values of Long et al. [1993] determined for gypsum in the $>5 \mu \mathrm{m}$ region, as described in more detail below, and these are used to determine $n$ as a function of wavelength.

\section{Results/Discussion}

\subsection{Scattering Theory Results}

4.1.1. Hapke Models

[27] The ASTER reflectance data for the $<45 \mu \mathrm{m}$ and $45-125 \mu \mathrm{m}$ grain size fractions of gypsum discussed in section 2.1 were used to determine the $k$ values. Using the $n$ values determined from the Fresnel reflectance discussed in section 4.2 and assuming a linear behavior of $n$ in the $0.4-$ $2.5 \mu \mathrm{m}$ region, a manual grid search was performed by varying the estimated grain size of the sample. At each point in the grid the $\chi^{2}$ value was calculated as an estimate of the goodness of the fit. Figure 5 is a shaded contour plot showing the resulting $\chi^{2}$ values. The two intersecting lines shown in Figure 5 indicate the final diameters associated with the lowest $\chi^{2}$ values. These diameters are $63.5 \mu \mathrm{m}$ for the $<45 \mu \mathrm{m}$ sample and $134.5 \mu \mathrm{m}$ for the $45-125 \mu \mathrm{m}$ sample. These final dimensions are problematic as they lie outside the range of the sieve opening sizes. As discussed in section 4.1.2, some particles larger than the sieve dimensions may be present in a sieved sample. However, we 


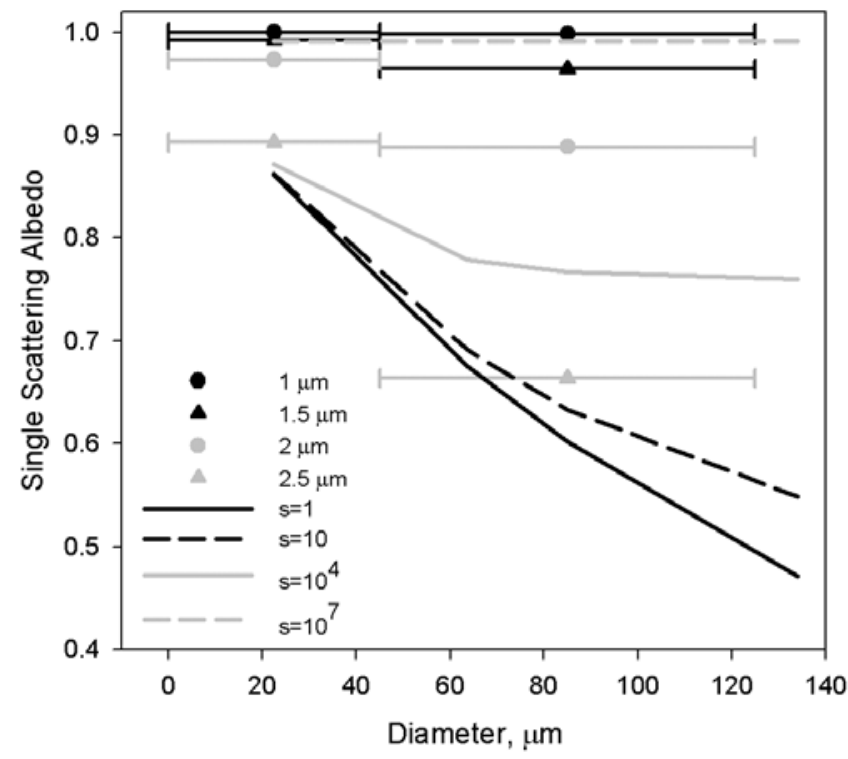

Figure 6. Single scattering albedo values calculated from the measured reflectance at several wavelengths in the nearinfrared (points) are compared to the single scattering albedos calculated for different grain diameters for different values of the internal scattering coefficient, $s$ (lines).

believe it would require the presence of too many such particles to create an average particle size of the sieved sample to be greater than the sieve dimensions. These samples are not in our possession, so we have no means of characterizing their particle size distribution.

[28] As discussed in section 3.1.1 in the standard approach, Hapke's internal scattering parameter, $s$, is set at $10^{-17}$ (units of $\mathrm{cm}^{-1}$ ). Little is known about this parameter for natural samples, but Lucey [1998] discusses this issue and shows some results that support his use of a particular value of $s$ for his analyses. At a single wavelength, Lucey [1998] compared the single scattering albedo (ssa) obtained from the measured reflectance of various grain sizes to trends predicted for the ssa resulting from calculations using differing values of $s$ [Lucey, 1998, Figures 1a and 1b].

[29] Here an analysis of varying the value of $s$ on the derived diameters was performed. First, the reflectance was calculated, along with the associated ssa, using the optical constants, derived in the following section, for 4 different grain sizes $(22.5,63.5,85$, and $134.5 \mu \mathrm{m})$. This calculation was performed for values of $s$ of $0,10^{-5}, 10^{-2}, 1,10,10^{4}$, and $10^{7}$. Independently the ssa is calculated directly from the laboratory reflectance data using equation (11.6) of Hapke [1993]. There is no assumption about grain size in this calculation and it was applied to the fine and medium grain spectra from the ASTER library. Lucey [1998] does not discuss that $s$ may be a function of wavelength. This issue is illustrated in Figure 6 where the data points represent the ssa derived from the reflectance measurements and the lines the trends associated with calculated reflectance of different grain sizes. The ssa in regions of weak absorption $(\sim 1 \mu \mathrm{m})$ require a high value of $s$ to reproduce the trend associated with the ASTER data. In the regions of stronger absorption (also increasing with wavelength) the trend associated with the ASTER data require increasingly lower values of $s$. Clearly a more detailed study of the wavelength behavior of $s$ is warranted, but beyond the scope of the current paper.

[30] Using a manual investigation of varying $s$, we find an $s$ value of $1.25 \mathrm{~cm}^{-1}$, and median diameters for the fine $(22.5 \mu \mathrm{m})$ and medium $(85 \mu \mathrm{m})$ grain size ASTER reflectance spectra, respectively, allows derivation of $k$ values that are consistent with each other and are comparable to those of Long et al. [1993] except at the highest $k$ values. These results are shown in Figure 7 where they are compared to the Long et al. [1993] averages and $k$ values derived via the Shkuratov model, discussed in the next section, where no parameter equivalent to $s$ exists.

[31] The hatched boxes in Figure 7 illustrate that for regions of high $k$ values neither scattering theory provides a reasonable comparison to those of Long et al. [1993], although the Hapke approach appears to provide better estimated values than the Shkuratov approach. When $k$ is large the measured diffuse reflectance is so low that $k$ eventually reaches a value where the calculated reflectance is no longer sensitive to changes in $k$ during the fitting procedure. In contrast, this same region is where the derivation of $k$ from Fresnel measurements is the most sensitive. This suggests that in the regions of strong absorption Fresnel measurements are the preferred approach.

[32] The cross-hatched box in Figure 7 illustrates that both scattering theories are sensitive to the band in the $2000-2500 \mathrm{~cm}^{-1}$ region where $k$ values are relatively low. In contrast, when $k$ is small the derivation of $k$ from Fresnel measurements is the least sensitive. This suggests that in the regions of weak absorption the scattering theories provide a better approach to determining $k$ values.

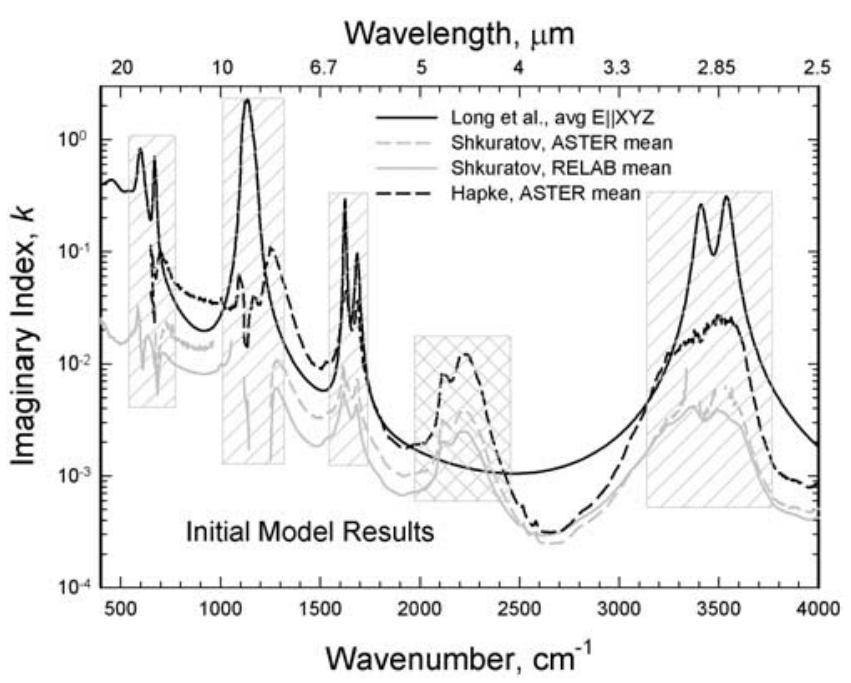

Figure 7. Comparison of $k$ values derived via Hapke [1993], for the ASTER library reflectance data (dashed black line), and Shkuratov et al. [1999], for the ASTER (dashed gray line) and RELAB (solid gray line) reflectance data, theories compared to the average values of Long et al. [1993] (solid black line). Both scattering theories have difficulty in determining $k$ for large values (gray hatched boxes). However, the Long et al. [1993] data do not accurately account for $k$ values near $2000-2500 \mathrm{~cm}^{-1}$ (gray cross-hatched box). 
Table 2. Parameters Associated With Initial Shkuratov Model Calculations

\begin{tabular}{|c|c|c|c|c|}
\hline \multirow[b]{2}{*}{ Reflectance Data } & \multirow{2}{*}{$\begin{array}{l}\text { Initial Diameters, } \\
\mu \mu \mathrm{m}\end{array}$} & \multicolumn{2}{|c|}{ Diameter Limits, $\mu \mathrm{m}$} & \multirow{2}{*}{$\begin{array}{c}\text { Diameter After } \\
\text { Minimization, } \mu \mathrm{m}\end{array}$} \\
\hline & & Lower & Upper & \\
\hline ASTER $<45 \mu \mathrm{m}$ & 22.5 & $1 \times 10^{-6}$ & 60.0 & 60.0 \\
\hline ASTER $45-125 \mu \mathrm{m}$ & 85.0 & 45.0 & 150.0 & 112.1 \\
\hline ASTER $125-500 \mu \mathrm{m}$ & 187.5 & 125.0 & 520.0 & 125.0 \\
\hline RELAB $<45 \mu \mathrm{m}$ & 22.5 & $1 \times 10^{-6}$ & 60.0 & 47.0 \\
\hline RELAB $25-75 \mu \mathrm{m}$ & 50. & 25.0 & 90.0 & 90.0 \\
\hline RELAB $63-90 \mu \mathrm{m}$ & 76.5 & 63.0 & 110.0 & 63.0 \\
\hline
\end{tabular}

\subsubsection{Shkuratov Models}

[33] Initial derivation of the optical constants using the Shkuratov et al. [1999] model involved both the ASTER and RELAB measurements. As described in section 3.1.2, Shkuratov's model is invertible, yielding $k$ starting from the bidirectional reflectance, and knowledge or estimates regarding the real index $n$, and porosity $q$, and average grain size $S$. Neither the ASTER nor RELAB samples are characterized in terms of size distribution of the samples. So, for each series of laboratory data, we performed a nonlinear fit, adjusting the value of each average size in order to minimize the deviations among the values of $k(\lambda)$ obtained. We assumed $q=0.7$, recognizing that variation in porosity does not significantly affect the reflectance [Shkuratov et al., 1999]. Similar to the approach described in section 4.1.1, a minimization of the difference between the measured and calculated reflectance is used. The approach specifically used $n$ values determined from the Fresnel reflectance for $\lambda<2.5 \mu \mathrm{m}$ and assuming a linear behavior of $n$ in the visible and near-infrared region. The fit procedure has been extended to the entire spectral range covered by the laboratory data used.

[34] Table 2 provides the details of the initial input parameters, constraints imposed on these, and the final results from the fitting procedure. Upper limits greater than the highest value of the sieve range size are used because, on the basis of laboratory experience, elongated particles can pass through the grid of a sieve in a length-wise direction, even if the long axis of the particle is larger than the opening in the grid. The mean values of the results for the data from each spectral library are shown in Figure 7. Inspection of Figure 7 shows that the resulting $k$ values are very similar for the data from the different spectral libraries. In contrast, the resulting $k$ values are lower then previous estimations for gypsum, obtained via dispersion analysis, by up to an order of magnitude. Like the results from section 4.1.1, comparison of $k$ values with previous estimates, obtained from dispersion analysis, suggests that the application of Shkuratov model in a region of strong absorptions does not work properly. In fact, in most relations it is assumed that $k<10^{-2}$ [Shkuratov et al., 1999]. Moreover, in regions of strong absorption, the parameter $S$ no longer approximates the average size of particles.

[35] The difference in absolute values between $k$ values obtained from the scattering theory and from dispersion analysis is probably due to an ambiguous determination of $S$. This is also evidenced by the final $S$ values obtained with the fit procedure, which are very often equal to one of the imposed diameter limits. As dispersion analysis is not influenced by this unknown parameter, we decided to force the fit procedure to search for values of grain size which minimize the deviations among $k$ values and simultaneously minimize the distance between these values and the results of dispersion analysis in the spectral ranges where $k$ values are $<0.01$ (transparent regions). This approach is commonly referred to as a penalty function method [Frodesen et al., 1979]. The region around $2200 \mathrm{~cm}^{-1}$ has not been considered as it is not well represented in the Long et al. [1993] data.

[36] The application of this method to RELAB and ASTER data is reported in Figure 8. RELAB data seem to fit better Long et al. data. Moreover, the obtained size values are now inside the imposed limits being: (10.08 \pm $0.01) \mu \mathrm{m}$ for the fine-grained sample $(<45 \mu \mathrm{m})$ and $(28.84 \pm$ $0.02) \mu \mathrm{m}$ for the medium-grain size sample $(25-75 \mu \mathrm{m})$.

\subsection{Transmission}

[37] The transmission of the gypsum samples was measured with the sample chamber "scrubbed" for $\mathrm{CO}_{2}$ and $\mathrm{H}_{2} \mathrm{O}$. This suggests that $\mathrm{m}_{0}$ associated with equation (1) is the complex refractive index of air. To illustrate the use of equations (1)-(3), we fix $n$ of gypsum to 1.531 , the highest value listed by Deer et al. [1966]. We use the measured

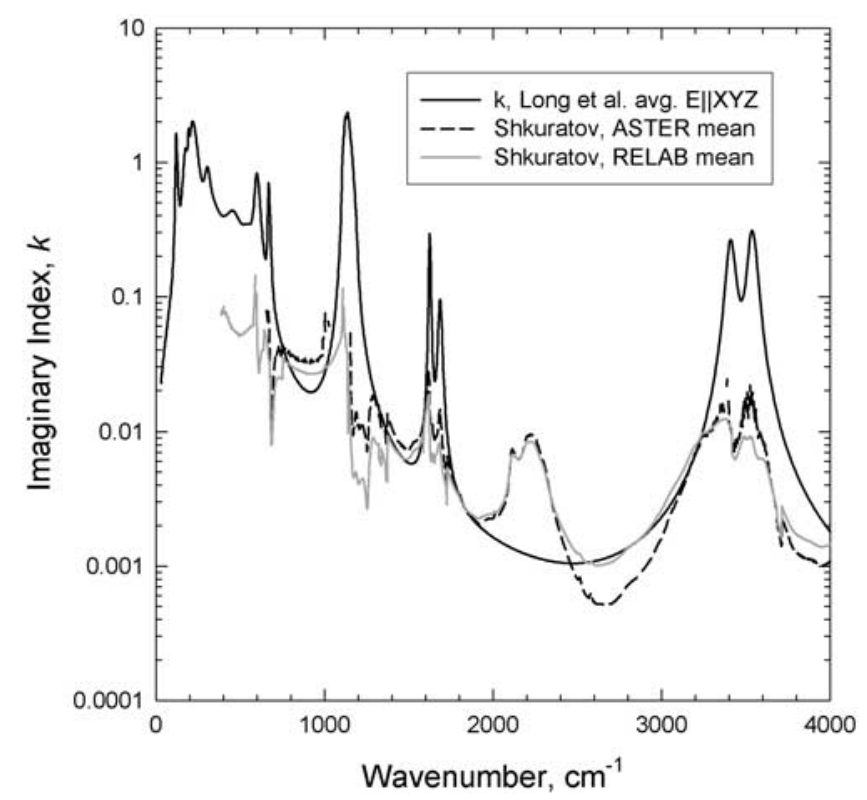

Figure 8. The $k$ values derived via Shkuratov et al. [1999] theory for the ASTER library reflectance data (dashed black line) and RELAB (solid gray line) reflectance data, using the penalty function method. Values obtained are compared to the average values of Long et al. [1993] (solid black line). 

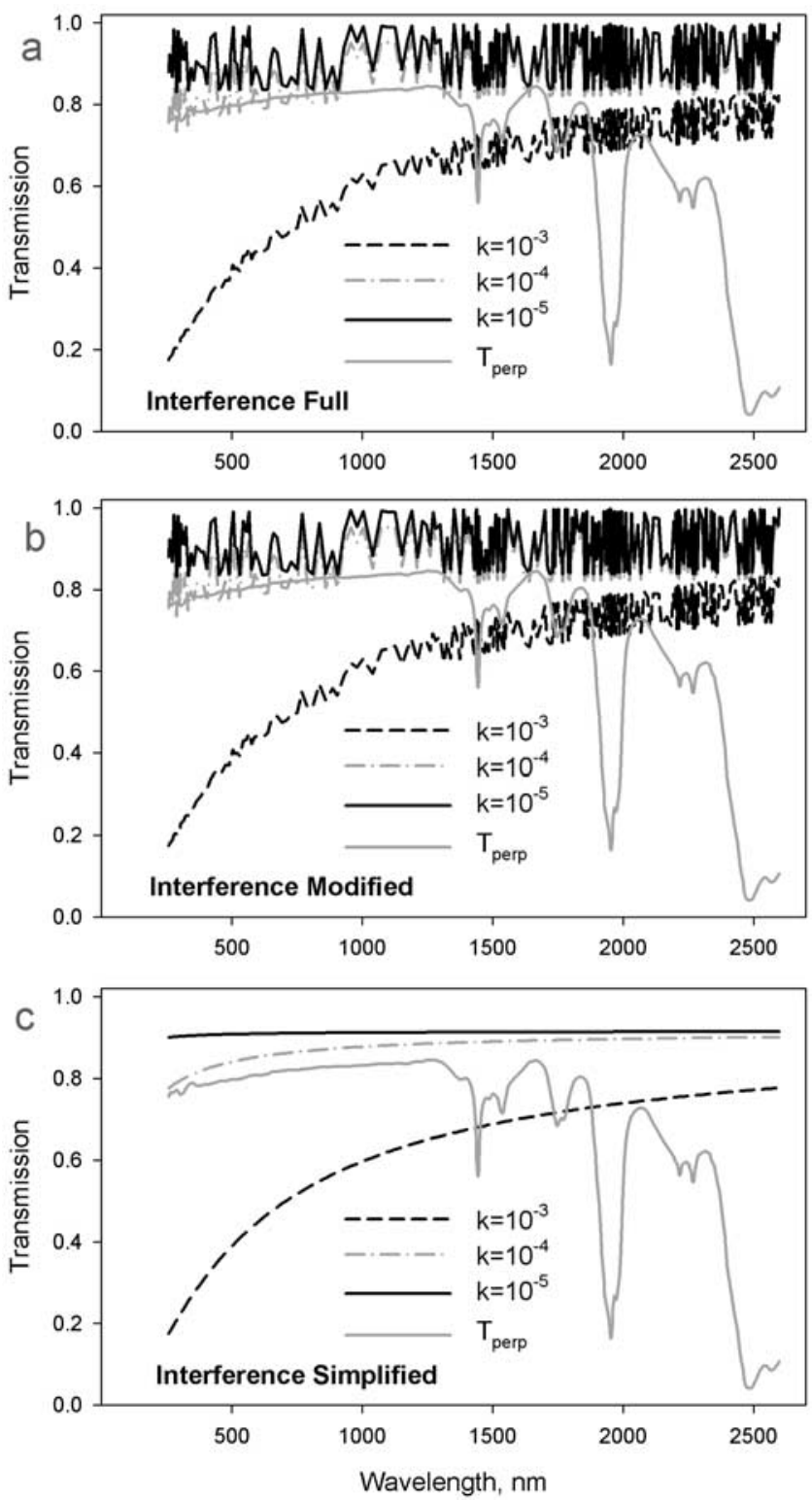

Figure 9. Transmission spectrum of face perpendicular to $\{010\}$ crystal face (dark heavy gray line) compared to calculated spectra using the (a) full, (b) modified, and (c) simplified models of transmission through a slab. Each panel shows various constant $k$ values for the slab. It is clear that the measured spectrum does not contain any of the high-frequency fringing as predicted by the full and modified models.

thickness of the thinnest sample $(0.034 \mathrm{~mm})$ and then fix $k$ of gypsum at several values. Even though we have concerns about scattering within this sample, it provides an opportunity to evaluate which of the equations above best describe the measurements.

[38] Calculations of the slab transmission using equation (1) for the thinnest sample are shown in Figure 9a. The different curves illustrate that the effect of increasing $k$ is to reduce the amplitude of the interference fringes at all wavelengths and preferentially reduce the transmission at the shorter wavelengths. The wavelengths of the maxima can be predicted by considering the interference phenomenon. Transmission maxima occur when $\zeta=$ $2 \pi \mathrm{p}$, where $\mathrm{p}=1,2,3, \ldots$. From section $3.3, \zeta=4 \pi \mathrm{n}_{1} \mathrm{~d} / \lambda$. Combining these expressions and canceling like terms yields $\lambda=2 \mathrm{n}_{1} \mathrm{~d} / \mathrm{p}$. For $\mathrm{p}$ values of $400,800,1600$ the wavelengths of the predicted maxima are 2.6, 1.3, and $0.65 \mu \mathrm{m}$, respectively. This illustrates that there are hundreds to thousands of maxima within the wavelength region of the transmission measurements. The calculations, for the thinnest sample, using equation (2) are shown in Figure $9 \mathrm{~b}$. The effects of increasing $k$ within the slab are identical to the trend seen in Figure 9a. Finally, the results of calculations using equation (3), i.e., ignoring the oscillatory term, are shown in Figure 9c for the thinnest sample. Similar to Figures $9 \mathrm{a}$ and $9 \mathrm{~b}$, effect of increasing $k$ within the slab is to preferentially reduce the transmission at the shorter wavelengths. For this case, we also calculated the transmission using the real index of gypsum of 1.519 , the lowest value listed by Deer et al. [1966], for $\mathrm{k}_{1}=10^{-4}$. The results are indistinguishable from the values using 1.531, and are not shown here.

[39] The solid heavy gray line in Figures $9 a-9 c$ is the transmission measured with the light beam polarized perpendicular to the $\{010\}$ plane of the sample. There is little evidence for the oscillatory interference fringes the theoretical calculations would predict. As a result we use equation (3) to derive the initial estimates of $n$ and $k$ for gypsum from the thinnest sample. We revisit this decision later in light of the limited mathematical experiment presented here.

[40] We investigated the potential source of errors associated with our assumption $\mathrm{n}_{\text {air }}$ was constant. At each wavelength the sum of the squared difference between the measured transmission $\left(\mathrm{T}_{\mathrm{m}}\right)$ and $\mathrm{T}_{\text {slab, }}$ from equation (3), was used to calculate an effective $n$ and $k$ for gypsum. We began each fit assuming $n$ for gypsum was a constant value of 1.523 that falls within the range given by Deer et al. [1966]. Our initial estimates of $k$ for gypsum came from applying a simple Beer-Lambert law to $\mathrm{T}_{\mathrm{m}}, \mathrm{T}_{\mathrm{m}}=\mathrm{e}^{-4 \pi \mathrm{kt} / \lambda}$, where $t$ is the sample thickness and $\lambda$ is in the same units as t. We used the AMOEBA algorithm contained within the Interactive Data Language (IDL $\left.{ }^{(}\right)$for our efforts. This algorithm uses a downhill simplex method as discussed in more detail by Press et al. [1992]. There are several parameters controlling the algorithm. These include defining the initial starting location of the simplex and a tolerance criterion $\left(f_{\text {tol }}\right)$ used to terminate the fitting procedure. The smaller the value of $f_{\text {tol }}$, the smaller the change is between successive values of the desired parameters, i.e., $n$ and $k$, within the minimization routine. Two convenient Web-based calculators are available to calculate $n_{\text {air }}$ for a range of wavelengths, and for numerous assumptions associated with the measurement conditions, e.g., temperature, pressure, relative humidity, etc. Using these, we find that for the ranges of temperature and pressure variations that might be associated with the laboratory measurements and for a relative humidity range of $0-100 \%$ the results were $1.00026<$ $n_{\text {air }}<1.00028$ over the entire wavelength range of the laboratory transmission measurements. We calculated the difference, $\Delta n_{\text {gypsum }}$, between separate results using values for $\mathrm{n}_{\text {air }}$ of $1.00026,1.00027$, and 1.00028 and found that $\left|\Delta n_{\text {gypsum }}\right|<3.4 \times 10^{-4}$. We conclude that achieving accuracy in the third decimal for $n_{\text {gypsum }}$ is independent of 
Table 3. Initial Estimates of $n_{\text {gypsum, }}$ Accuracy of Fit $\Sigma\left(T_{m}-\right.$ $\left.T_{\text {slab }}\right)^{2}$, and $n_{\text {gypsum }}$ Average $(\bar{n})$ for $\mathrm{T}_{\perp}$ to Crystal Striations

\begin{tabular}{lcc}
\hline$n_{\text {initial }}$ & $\Sigma\left(\mathrm{T}_{\mathrm{m}}-\mathrm{T}_{\text {slab }}\right)^{2} \times 10^{-30}$ & $\bar{n}$ \\
\hline 1.519 & 7.210 & 1.518 \\
1.521 & 1.239 & 1.520 \\
1.523 & 2.830 & 1.522 \\
1.525 & 2.111 & 1.524 \\
1.527 & 7.455 & 1.526 \\
1.529 & 8.159 & 1.528 \\
1.531 & 10.827 & 1.530 \\
\hline
\end{tabular}

the possible variations in $n_{\text {air }}$ at these wavelengths. As a result, we restrict further calculations to use $n_{\text {air }}=1.00027$.

[41] With $n_{\text {air }}$ fixed, we investigated the sensitivity of our results on the initial estimates for $n_{\text {gypsum. }}$. We perform this evaluation by using initial estimates for $n_{\text {gypsum }}$ of $1.519-$
1.531 , at an increment of every 0.002 . This range of values samples the extremes of those listed by Deer et al. [1966] for all optic axes. However, we note the range along an individual optic axis is less: $\sim 0.002$. Table 3 summarizes the results in terms of the differences between models and observations and also provides the average of $n_{\text {gypsum }}(\bar{n})$, at all wavelengths, determined from the analyses. The smallest difference value (Table 3 , row 2) corresponds to $n_{\text {init }}$ of 1.521 with an $\bar{n}$ of 1.520 . The resulting $n_{\text {gypsum }}$ and associated $k_{\text {gypsum }}$ are shown in Figures $10 \mathrm{~b}$ and $10 \mathrm{j}$, respectively. Using equation (3) we find that $f_{\text {tol }}=1 . \times$ $10^{-14}$ allows convergence to a solution. Upon convergence, the maximum peak-to-peak difference of the resulting $n_{\text {gypsum }}$ is $\sim 0.004$ and the maximum absolute difference between $\mathrm{T}_{\text {slab }}$ and $\mathrm{T}_{\mathrm{m}}$ is $\sim 4 \times 10^{-16}$.

[42] We evaluate whether the use of the simplified transmission expression of equation (3) might give rise to the
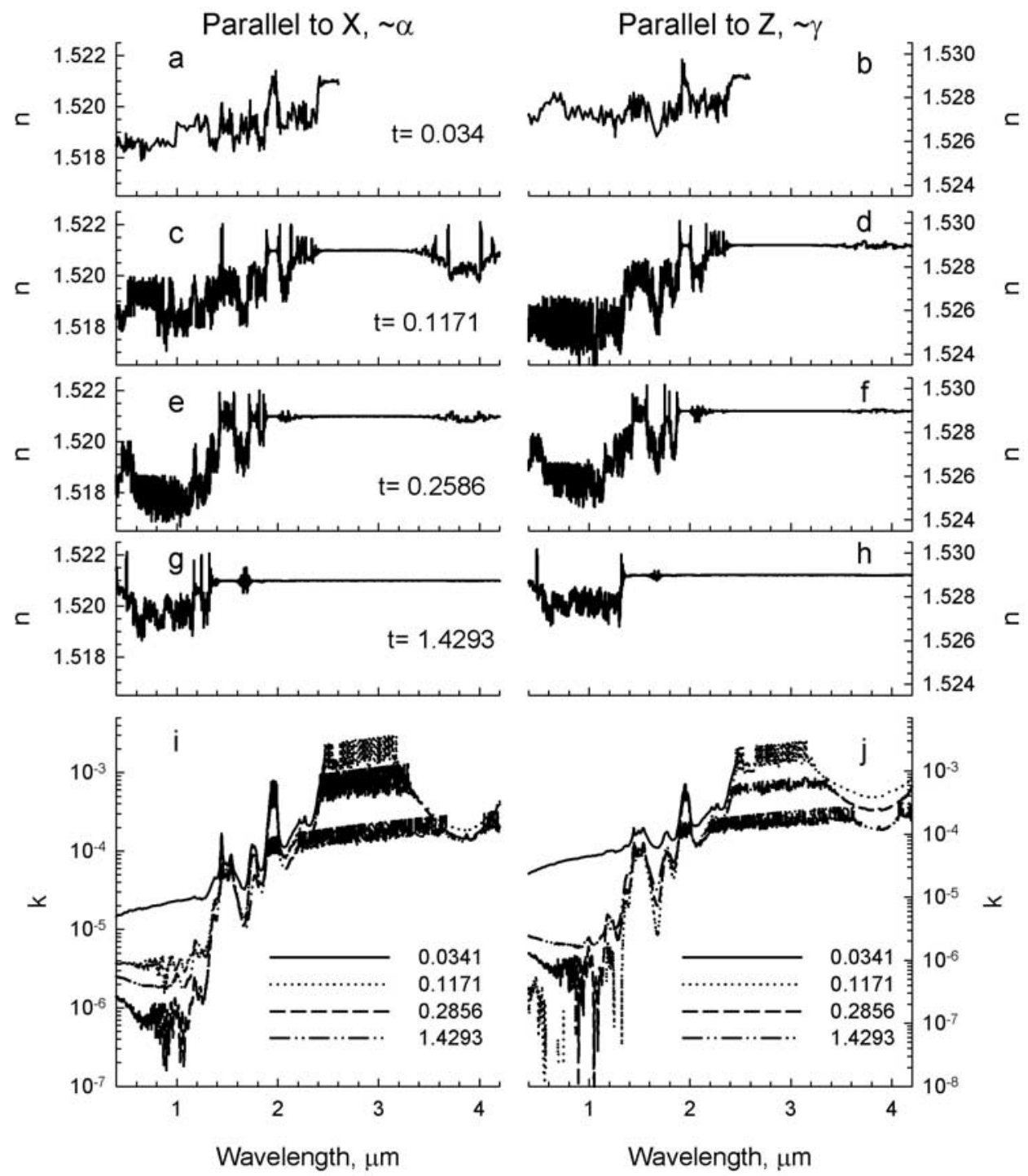

Figure 10. Optical constants of different gypsum samples for polarizations of the incident beam relative to the crystal axes. Derived real index of refraction for various sample thicknesses: (a and b) the sample thickness is $0.034 \mathrm{~cm}$, (c and d) the sample thickness is $0.1171 \mathrm{~cm}$, (e and f) the sample thickness is $0.2586 \mathrm{~cm}$, and $(\mathrm{g}$ and $\mathrm{h}$ ) the sample thickness is $1.4293 \mathrm{~cm}$. ( $\mathrm{i}$ and $\mathrm{j}$ ) Derived imaginary index of refraction for the various thickness samples. 
Table 4. Initial Estimates of $n_{\text {gypsum, }}$ Accuracy of Fit $\Sigma\left(T_{m}-\right.$ $\left.T_{\text {slab }}\right)^{2}$, and $n_{\text {gypsum }}$ Average $(\bar{n})$ for $\mathrm{T}_{\|}$to Crystal Striations

\begin{tabular}{lcc}
\hline$n_{\text {initial }}$ & $\Sigma\left(\mathrm{T}_{\mathrm{m}}-\mathrm{T}_{\text {slab }}\right)^{2} \times 10^{-30}$ & $\bar{n}$ \\
\hline 1.519 & 102.6 & 1.518 \\
1.521 & 2.143 & 1.520 \\
1.523 & 17.74 & 1.522 \\
1.525 & 2.409 & 1.524 \\
1.527 & 7.527 & 1.526 \\
1.529 & 2.012 & 1.528 \\
1.531 & 3.263 & 1.530 \\
\hline
\end{tabular}

relatively narrow peak-to-peak variability seen in Figure $10 \mathrm{~b}$. We do so by using $\mathrm{n}_{\text {air }}=1.00027$ and $\mathrm{n}_{\text {init }}=1.521$ and use these in equations (1) and (2) and evaluate if the observed variability seen in Figure $10 \mathrm{~b}$ is eliminated.

[43] Using equation (2), we find that $f_{\text {tol }}=0.75$ is required to achieve convergence. Such a high tolerance value compared to the simple model is the first clue that the more complex model might not describe the laboratory data. Upon convergence we find a maximum peak-to-peak difference of $\sim 0.01$ for the resulting $n_{\text {gypsum }}$ and a maximum absolute difference between $T_{\text {slab }}$ and $T_{m}$ of $\sim 0.12$. The associated best fit parameter is 0.230 ; tens of orders of magnitude higher than all the values listed in Table 3.

[44] Using equation ( 1 ), we find that $f_{\text {tol }}=0.5$ is required to achieve convergence. Again, such a high tolerance value compared to the simple model is a clue that this more complex model might not describe the laboratory data. Upon convergence we find a maximum peak-to-peak dif- ference of $\sim 0.01$ for the resulting $n_{\text {gypsum }}$ and a maximum absolute difference between $T_{\text {slab }}$ and $T_{m}$ of $\sim 0.13$. The associated best fit parameter is 0.118 , again tens of orders of magnitude higher than the values listed in Table 3.

[45] For a more straightforward comparison between the models, we used equation (3) and set $f_{\text {tol }}=0.5$ where convergence is again achieved. Upon convergence we again find a maximum peak-to-peak difference of $\sim 0.004$ for resulting $n_{\text {gypsum }}$, about the same as before, and a maximum absolute difference between $\mathrm{T}_{\text {slab }}$ and $\mathrm{T}_{\mathrm{m}}$ of $\sim 0.06$, significantly worse than the $\sim 4 \times 10^{-16}$ achieved previously. The best fit parameter is 0.14 , significantly worse than the values listed in Table 3. In light of the facts that (1) the simple model can converge to a solution with much smaller tolerance parameter and (2) the resulting differences between the measured and modeled data are much smaller, we conclude that the laboratory measured transmission is best described by the simple model without accounting for the oscillatory fringe effects included in the more complex models. Thus we rely upon equation (3) for derivation of the final $k_{\text {gypsum }}$ over the wavelength range of the transmission measurements. As described below we determine $n_{\text {gypsum }}$ via an iterative approach using the Kramers-Kronig relationship.

[46] We now evaluate the transmission of the crystal where the incident beam is polarized parallel to the $\{010\}$ crystal face for the thinnest sample. The results of applying equation (3) while varying $n_{\text {init }}$ are shown in Table 4 . The results indicate the best fit value occurs with $\mathrm{n}_{\text {init }}=1.529$, although there is little difference in the results for $\mathrm{n}_{\text {init }}=$ 1.521. The beam polarized parallel to the $\{010\}$ crystal face has the potential to sample two separate optic axes with

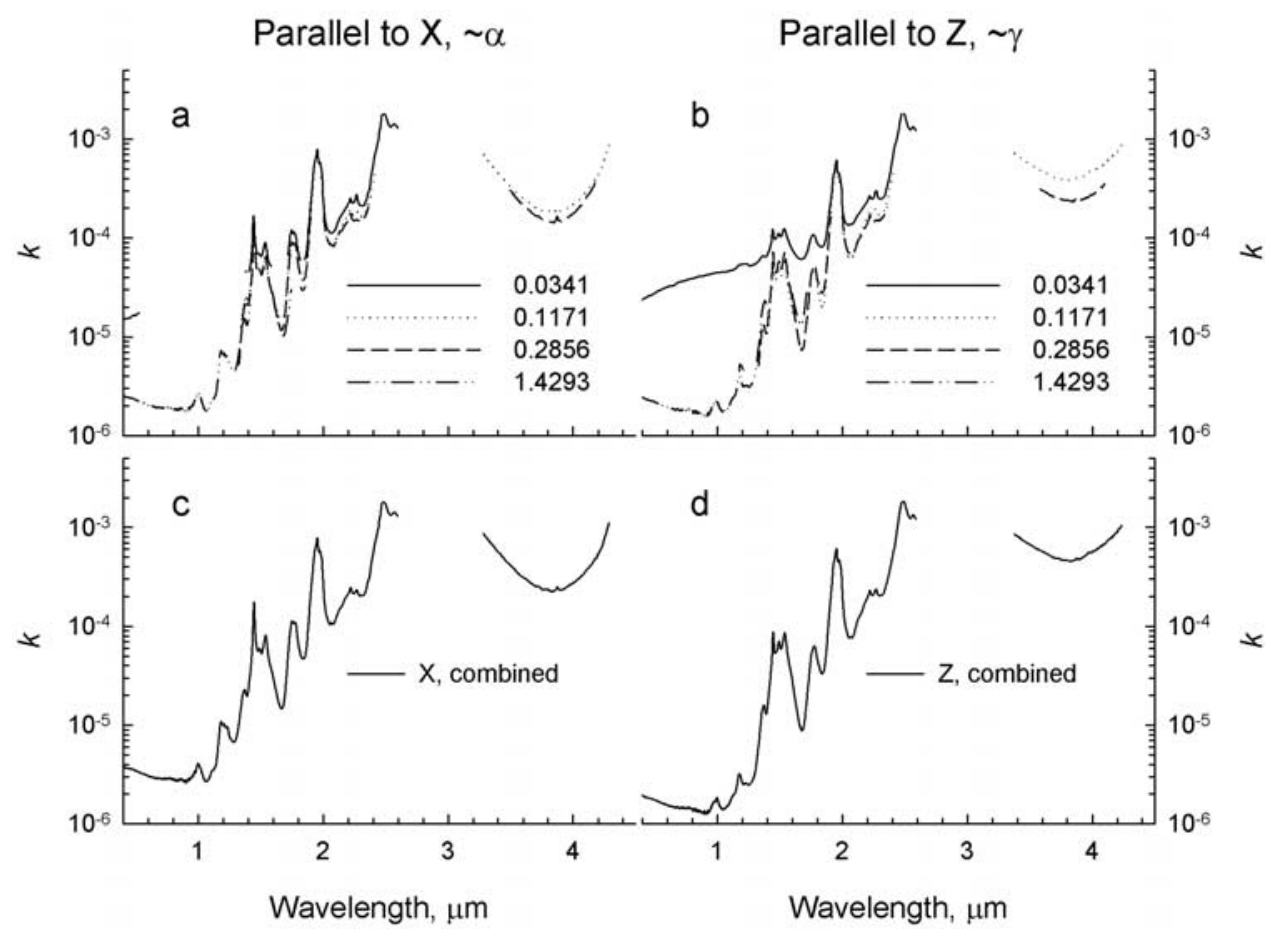

Figure 11. ( $a$ and $b$ ) Derived imaginary index of refraction for different polarizations of the incident beam relative to the crystal axes (left and right columns) and various sample thicknesses. (c and d) Combined imaginary index of refraction for each polarization. See text for details of how the data in Figures $11 \mathrm{a}$ and $11 \mathrm{~b}$ were combined to create the data in Figures 11c and 11d. 
Table 5. Data Used to Create Combined Imaginary Indices

\begin{tabular}{|c|c|c|c|}
\hline \multicolumn{2}{|c|}{ Parallel to $\mathrm{X}$} & \multicolumn{2}{|c|}{ Parallel to Z } \\
\hline Wavelength Range, $\mu \mathrm{m}$ & $k$ Values Used, cm & Wavelength Range, $\mu \mathrm{m}$ & $k$ Values Used, $\mathrm{cm}$ \\
\hline $0.35-1.415$ & $1.5 \times 1.4293$ & $0.35-1.3535$ & $0.8 \times 1.4293$ \\
\hline $1.415-1.7999$ & $1.25 \times 0.2856$ & $1.3539-1.9071$ & $1.2 \times 0.2856$ \\
\hline $1.8005-1.927$ & $1.25 \times 0.1171$ & $1.9078-1.9378$ & $1.2 \times 0.1171$ \\
\hline $1.9285-1.9818$ & unscaled 0.034 & $1.9410-1.9678$ & unscaled 0.034 \\
\hline $1.9830-2.3495$ & $1.25 \times 0.1171$ & $1.9679-2.4141$ & $1.2 \times 0.1171$ \\
\hline $2.3498-2.5982$ & unscaled 0.034 & $2.4532-2.5876$ & unscaled 0.034 \\
\hline $3.2840-4.2855$ & $1.25 \times 0.1171$ & $3.3760-4.2330$ & $1.2 \times 0.1171$ \\
\hline
\end{tabular}

associated $n$ values of 1.519-1.521, and 1.529-1.531, respectively [Deer et al., 1966]. Here we realize the measurements may actually be sampling contributions from each axis but we defer to the absolute accuracy minimum to define the $\mathrm{n}_{\text {init }}$ for use in determining $\mathrm{n}_{\text {gypsum }}$ and $\mathrm{k}_{\text {gypsum }}$ and the resulting values are shown in Figures 10a and 10i, respectively.

[47] Figures 10c, 10e, and 10g show the values determined for the real index of the thicker samples with the beam polarized approximately parallel to X, while Figures 10d, $10 \mathrm{f}$, and $10 \mathrm{~h}$ show these values for the beam polarized approximately parallel to Z . Figures $10 \mathrm{i}$ and $10 \mathrm{j}$ show the associated $k$ values for the beam polarized approximately parallel to $\mathrm{X}$ and $\mathrm{Z}$, respectively. As can be seen when absorption bands become strong enough, i.e., saturated, the derived real index is fairly constant and the associated imaginary index cannot be determined accurately.

[48] For each polarized measurement we wish to combine the derived imaginary indices shown in Figures $10 \mathrm{i}$ and $10 \mathrm{j}$. Transmission measurements in excess of $\sim 80 \%$ can result in inaccurate absorption measurements due to random and systematic errors and lead to large errors in the derived imaginary index [Irvine and Pollack, 1968; Warren, 1984; Hansen, 1997]. So, we eliminate any data where the transmittance is $>0.8$. Inaccuracies in estimates of the imaginary indices can also be introduced at low transmission values [Irvine and Pollack, 1968]. So, we arbitrarily eliminate any data where the transmittance $<0.04$. The data
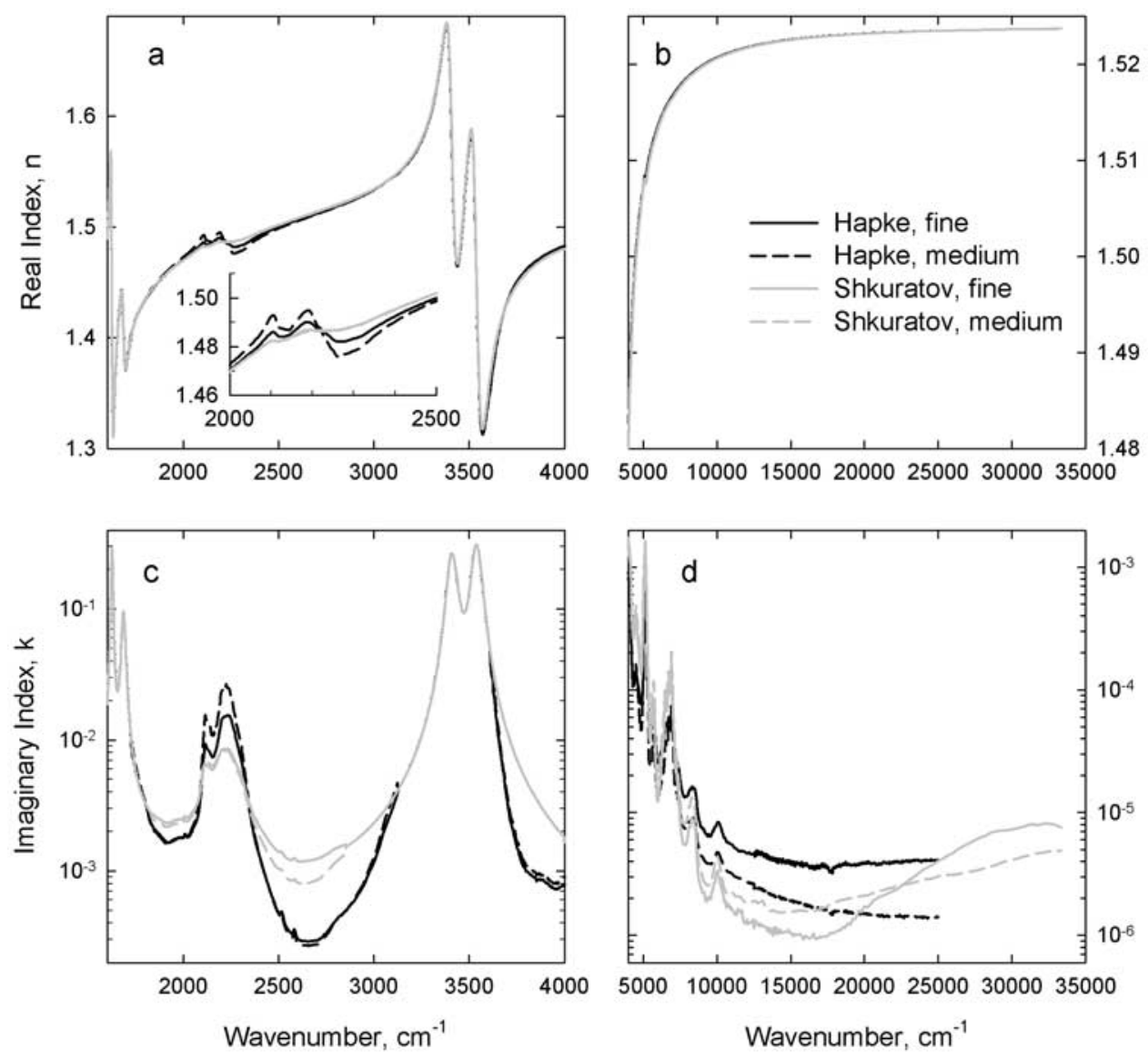

Figure 12. (a) The $n$ values derived via scattering theories in the infrared. An expanded view of the $2000-2500 \mathrm{~cm}^{-1}$ region is shown in the inset. (b) The $n$ values derived via scattering theories in the visible and near-infrared. (c) The $k$ values derived via scattering theories in the infrared. (d) The $k$ values derived via scattering theories in the visible and near-infrared. 

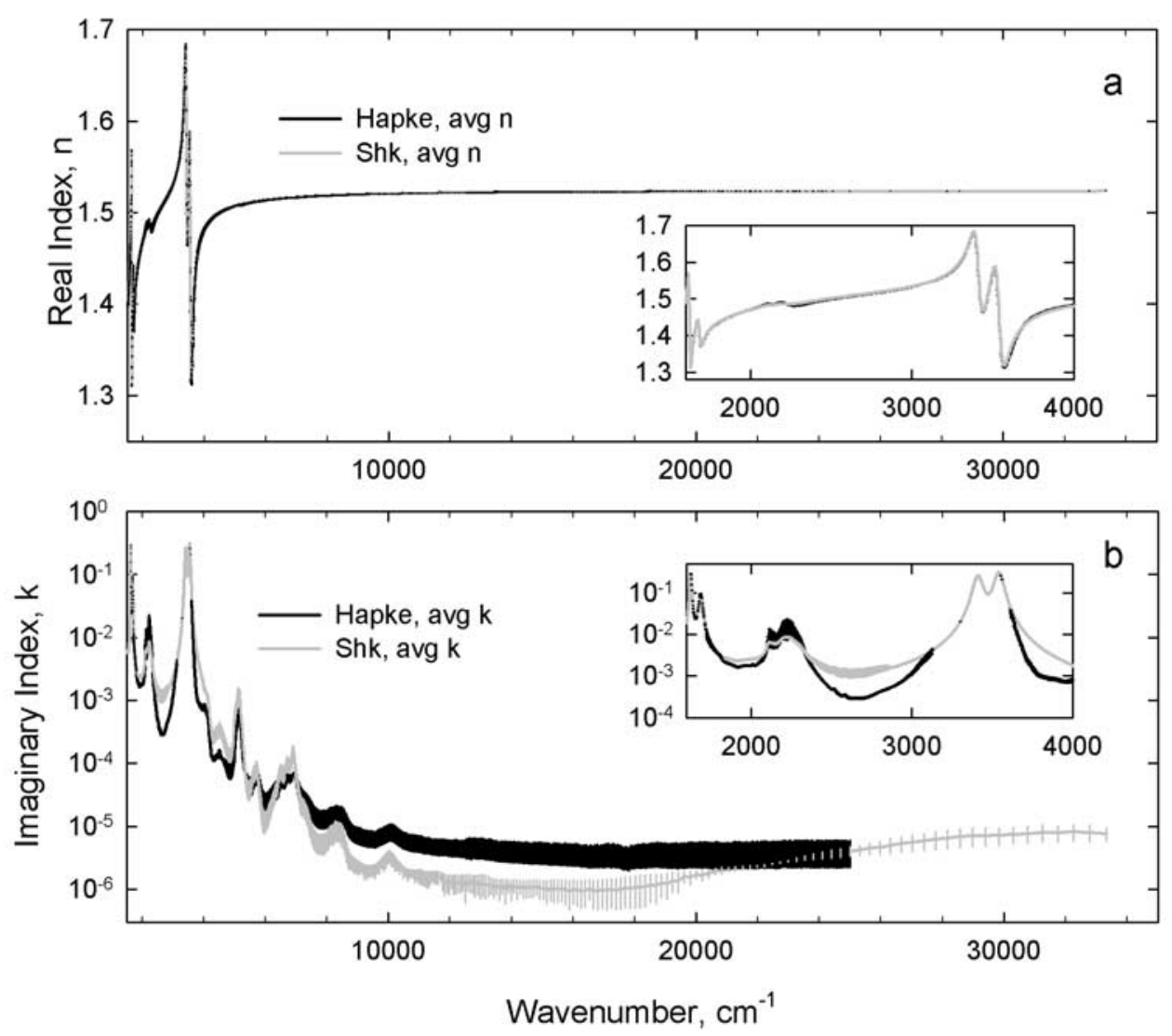

Figure 13. (a) Average $n$ values determined via Hapke (black line) and Shkuratov (gray line) scattering theories are shown with one standard deviation error bars. An enlargement of the infrared region is shown in the inset. (b) Average $k$ values determined via Hapke (black line) and Shkuratov (gray line) scattering theories are shown with one standard deviation error bars. An enlargement of the infrared region is shown in the inset.

remaining after application of these criteria are shown in Figures $11 \mathrm{a}$ and $11 \mathrm{~b}$.

[49] We note that only the thinnest sample has data near the $1.9 \mu \mathrm{m}$ maximum in $k$ values that meets the minimum transmittance criteria. Because the absorption is sufficiently high we believe that scattering is unimportant at these wavelengths and we use the $k$ values of the thinnest sample in the $1.9 \mu \mathrm{m}$ region to scale the other data in regions of overlap. We use this approach for both polarization measurements. In general, our philosophy is to use the thickest sample to estimate the $\mathrm{k}$ values. However, when the two intermediate thickness samples yield essentially equivalent results we may extend the range where the thinner sample results are used for consistency. The specifics about the scaling factors, and regions of wavelengths combined are provided in Table 5 for each polarization measurement and the resulting combined data are shown in Figures 11c and 11d.

\subsection{Compilation of $n$ and $k$}

[50] Following the approach described by Roush [2003, 2005], we used an iterative scattering theory-SK-K analysis to determine $n$ and $k$ from the various reflectance measurements. In this iterative process, the $k$ values derived from both scattering theory calculations were combined with the Long et al. [1993] data prior to the SK-K determination of $n$. At $<1836$ and $2856-4000 \mathrm{~cm}^{-1}$ the $k$ values of Long et al. [1993] are used, and between 1836 and $2856 \mathrm{~cm}^{-1}$ the $k$ values derived via the scattering theories are used. These combined $k$ values are used in the SK-K analysis to estimate $n$ values. This process is iterated until changes in $n$ and $k$ are insignificant and the results for the four different samples are shown in Figure 12. An average of the values derived from each grain size of the ASTER and RELAB data was calculated and these results are shown in Figure 13, along with their standard deviation. Finally, an average of all the results was calculated and is shown in Figure 14. The maximum standard deviation of the average $n$ is about 0.01 and the average $k$ about 0.02 . However, over much of the wavelength domain, these values are much lower, about $6 \times 10^{-4}$ for $n$ and $3 \times 10^{-4}$ for $k$.

[51] Figure 15a shows the $k$ values determined from transmission for each polarization in the visible and midinfrared $\left(0.33-5 \mu \mathrm{m}, 2000-30000 \mathrm{~cm}^{-1}\right)$. Because we wish to compare to the $k$ values derived from the reflectance measurements, where the samples are likely randomly oriented we average the $k$ values from the transmission measurements. The resulting average is compared to the average $k$ values derived via scattering theories in Figure $15 \mathrm{~b}$. In order to assess whether or not the modest differences seen in Figure 15b are significant we subsample both average values and plot the results along with the associated standard deviations in Figure $15 \mathrm{c}$, and an 

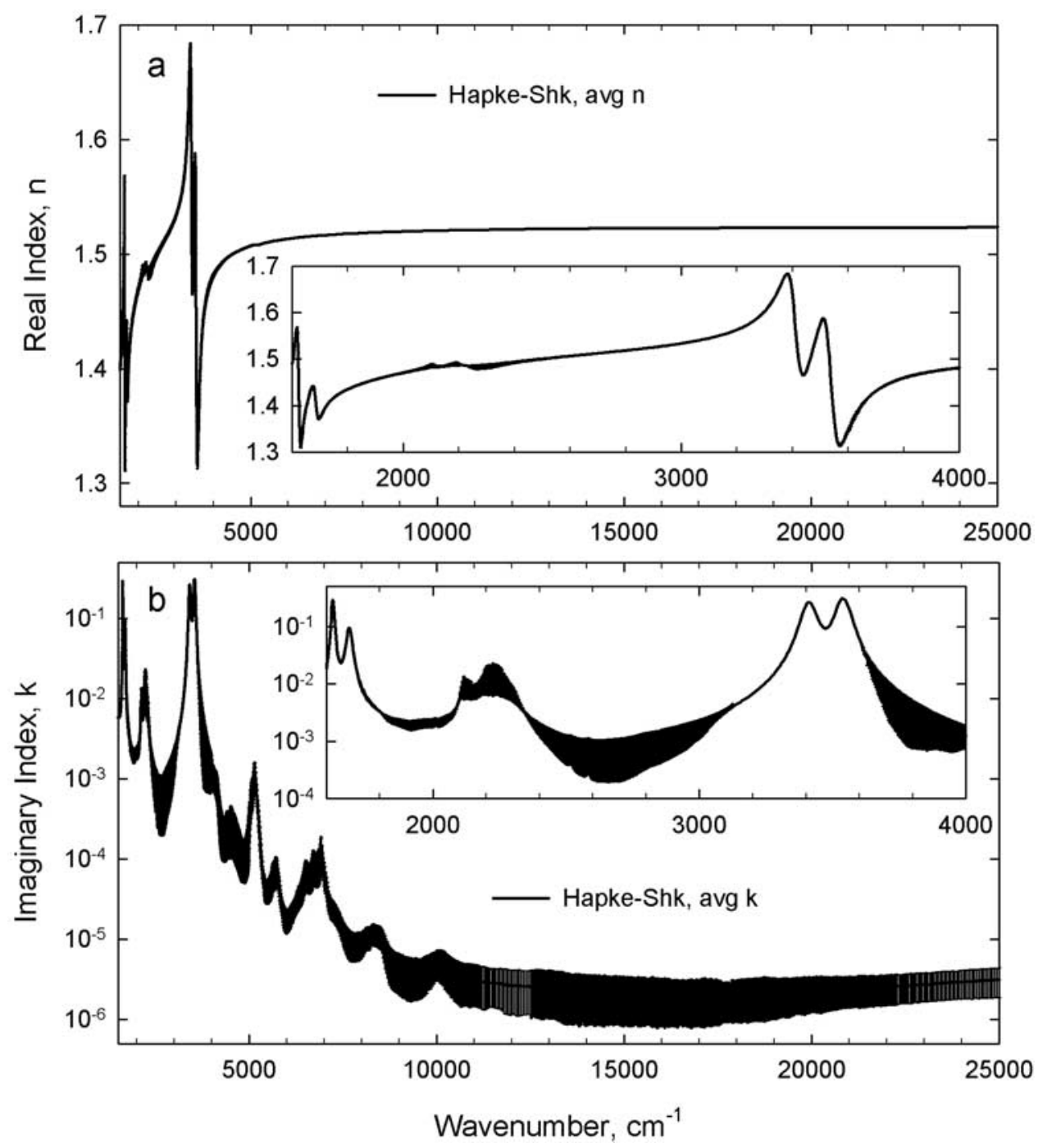

Figure 14. (a) Average of $n$ values from all scattering theory results and associated one standard deviation errors. An expanded view of the infrared region is shown in the inset. (b) Average of $k$ values from all scattering theory results and associated one standard deviation errors. An expanded view of the infrared region is shown in the inset.

enlargement of the $2300-8000 \mathrm{~cm}^{-1}$ region is shown in Figure $15 \mathrm{~d}$. It is clear that within one standard deviation the results are the same, with perhaps the exception at the lowest wave numbers, $<2400 \mathrm{~cm}^{-1}$. As a result, we use the existing compilation of $n$ and $k$ from the scattering calculations.

\section{Conclusions}

[52] We identify a discrepancy between the reported available infrared optical constants of gypsum and reflectance measurements of gypsum that clearly indicates an absorption near $4.5 \mu \mathrm{m}$. We conclude this discrepancy arises due to the relative weak nature of the $4.5 \mu \mathrm{m}$ feature that implies previous techniques were insensitive to its presence.

[53] We apply two different scattering theories to estimate the optical constants of gypsum in the visible, near-infrared, and infrared wavelengths. We conclude both of these theories are capable of addressing the weak features, but suffer from fundamental insensitivities where materials exhibit their highest $k$ values. Fortunately, this is exactly the opposite situation for optical constants determined via Fresnel reflectance measurements where they are sensitive when $k$ is high, but insensitive when $k$ is low. We recommend taking advantage of both techniques by applying them in the appropriate regions. This is especially true for samples where relatively thick and optically clear crystals are not readily available.

[54] We combine the results of the scattering theories with previous infrared results and calculate average $n$ and $k$ values and their associated standard deviations. We compare these with $k$ values estimated from transmission measurements at visible and short infrared wavelengths. We find the two derivations are in remarkable agreement. This supports the suggestion of Roush [2005] of combining the results of 

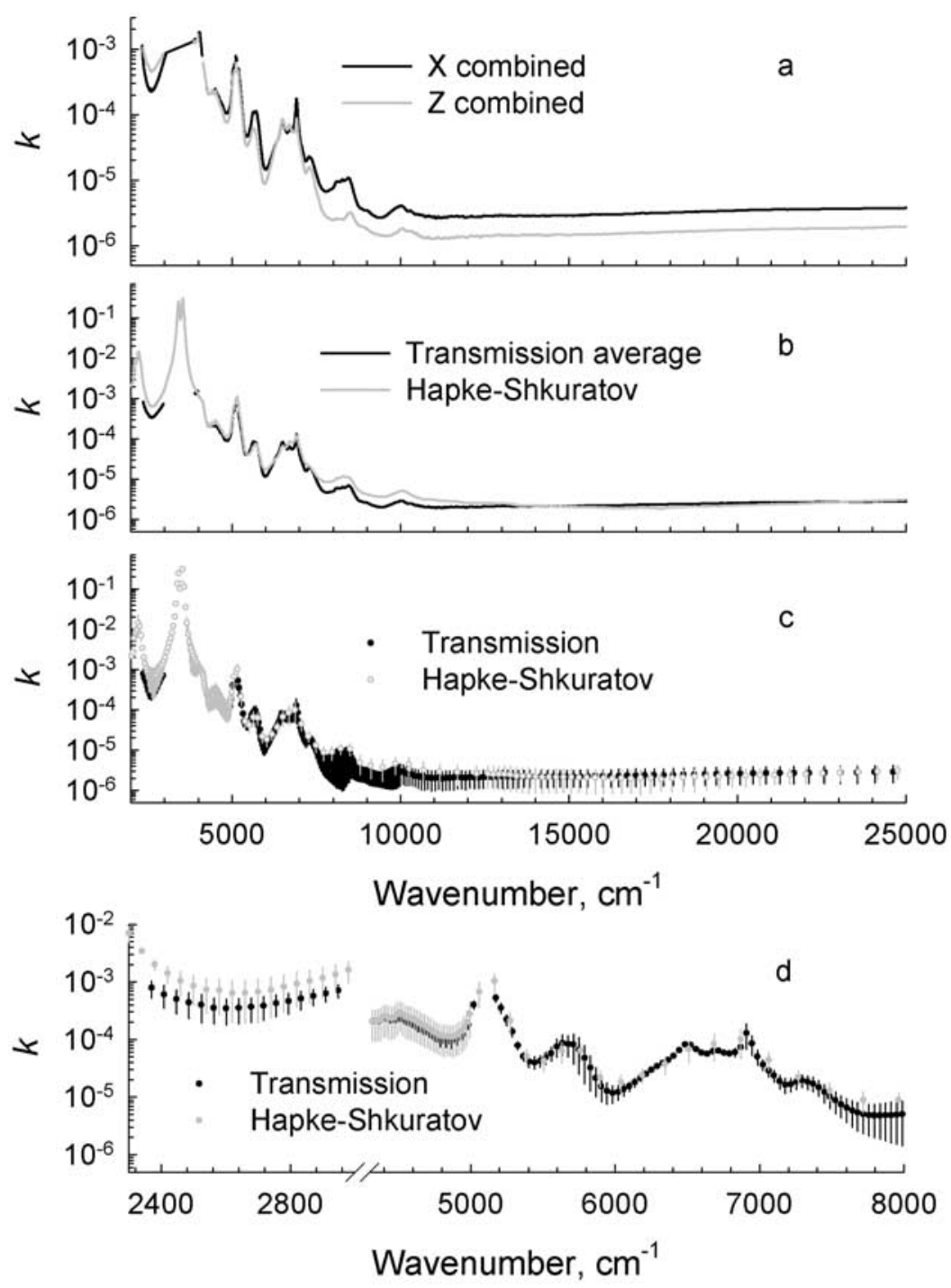

Figure 15. (a) Comparison of imaginary index of refraction along each polarization as a function of wave number. (b) Average polarization imaginary indices of refraction compared to the values derived from the scattering theory approach. (c) Subsampling of average data derived from transmission (black points with error bars) compared to subsampling of data derived from scattering approach (gray points with error bars). (d) Enlargement of the near-infrared region. In the region of overlap the estimated imaginary indices of refraction agree to within the standard deviations of the two data sets. 
scattering theories with Fresnel reflectance measurements provide more accurate estimates of the optical constants of materials.

[55] The average values of $n$ and $k$ shown in Figure 15 represent our best estimate of the optical constants of gypsum over the $30-25000 \mathrm{~cm}^{-1}$ and their uncertainties.

[56] Acknowledgments. We thank Giuseppe Marzo for providing the selenite sample used for the mid-infrared Fresnel measurements and a digital version of his derived optical constants. We extend our gratitude to Giuseppe Marzo and Edward Cloutis for their helpful reviews that improved the content of the original manuscript. T.L.R. recognizes the important support from NASA's Planetary Geology and Geophysics Program that enabled this research. F.E. and L.C. gratefully acknowledge funding from the Italian Space Agency (ASI) under contract I/010/05/0. G.R.R. acknowledges support from NASA's Mars Fundamental Research program.

\section{References}

Aronson, J. R., A. G. Emslie, V. Miseo, E. M. Smith, and P. F. Strong (1983), Optical constants of monoclinic anisotropic crystals: Gypsum, Appl. Opt., 22, 4093-4098.

Bevington, P. R. (1969), Data Reduction and Error Analysis for the Physical Sciences, 336 pp., McGraw-Hill, New York.

Bibring, J. P., et al. (2005), Mars surface diversity as revealed by the OMEGA/Mars Express observations, Science, 307, 1576-1581.

Blaney, D. L., and T. B. McCord (1995), Indications of sulfate minerals in the Martian soil from Earth-based spectroscopy, J. Geophys. Res., 100, $14,433-14,441$.

Bohren, C. F., and D. R. Huffman (1983), Absorption and Scattering of Light by Small Particles, 530 pp., John Wiley, New York.

Christensen, P. R., et al. (2004), Mineralogy at Meridiani Planum from the Mini-TES experiment on the Opportunity rover, Science, 306, 17331739.

Clark, R. N., and T. L. Roush (1984), Reflectance spectroscopy: Quantitative analysis techniques for remote sensing applications, J. Geophys. Res., 89, 6329-6340.

Coblentz, W. M. (1906), Investigations of infrared spectra. Part III. Infrared transmission spectra. Part IV. Infra-red reflection Spectra, Carnegie Inst. Washington Publ. 65, 128 pp.

Cruikshank, D. P., T. L. Roush, J. M. Moore, M. V. Sykes, T. C. Owen, M. J. Bartholomew, R. H. Brown, and K. A. Tryka (1997), The surfaces of Pluto and Charon, in Pluto and Charon, edited by S. A. Stern and D. J. Tholen, pp. 221-267, Univ. of Ariz. Press, Tucson.

Deer, W. A., R. A. Howie, and J. Zussman (1966), An Introduction to the Rock Forming Minerals, 528 pp., Longman, London.

Esposito, F., L. Colangeli, and E. Palomba (2000), Infrared reflectance spectroscopy of Martian analogues, J. Geophys. Res., 105(E7), $17,643-17,654$

Fink, U., and D. Burk (1971), Reflection spectra, 2.5-7 $\mu$, of some solids of planetary interest, Comm. Lunar. Planet. Lab. 185, pp. 8-20, Lunar and Planet. Lab., Tucson, Ariz.

Frodesen, A. G., O. Skjeggestad, and H. Tofte (1979), Probability and Statistics in Particle Physics, 372 pp., Columbia Univ. Press, New York. Gendrin, A., et al. (2005), Sulfates in Martian layered terrains: The OMEGA Mars Express view, Science, 307, 1587-1591.

Hansen, G. B. (1997), The infrared absorption spectrum of carbon dioxide ice from 1.8 to $333 \mu \mathrm{m}, J$. Geophys. Res., 102(E9), 21,569-21,587.

Hapke, B. (1981), Bidirectional reflectance spectroscopy: 1. Theory, J. Geophys Res. 96, 3039-3054.

Hapke, B. (1986), Bidirectional reflectance spectroscopy 4: Extinction and the opposition effect, Icarus, 67, 264-280.

Hapke, B. (1993), Theory of Reflectance and Emittance Spectroscopy, 455 pp., Cambridge Univ. Press, New York.

Hapke, B., and E. Wells (1981), Bidirectional reflectance spectroscopy 2: Experiments and observations, J. Geophys. Res., 96, 3055-3060.

Heavens, O. S. (1970), Thin Film Physics, 152 pp., Methuen, London.

Hovis, W. A., Jr. (1966), Infrared spectral reflectance of some common minerals, Appl. Opt., 5, 245-248.
Irvine, W. M., and J. B. Pollack (1968), Infrared optical properties of water and ice spheres, Icarus, 8, 324-360.

Klingelhöfer, G., et al. (2004), Jarosite and hematite at Meridiani Planum from Opportunity's Mössbauer Spectrometer, Science, 306, 1740-1745.

Langevin, Y., F. Poulet, J. P. Bibring, and B. Gondet (2005), Sulfates in the north polar region of Mars detected by OMEGA/Mars Express, Science, $307,1584-1586$

Long, L. L., M. R. Querry, R. J. Bell, and R. W. Alexander (1993), Optical properties of calcite and gypsum in crystalline and powdered form in the infrared and far-infrared, Infrared Phys., 34, 191-201.

Lucey, P. G. (1998), Model near-infrared optical constants of olivine and pyroxene as a function of iron content, J. Geophys. Res., 103(E1), 17031713

Marzo, G. A., A. Blanco, F. De Carlo, M. D’Elia, S. Fonti, A. C. Marra V. Orofino, and R. Politi (2004), The optical constants of gypsum particles as analog of Martian sulfates, Adv. Space Res., 33(12), 2246-2251.

Murchie, S., et al. (2003), CRISM: Compact Reconnaissance Imaging Spectrometer for Mars on the Mars Reconnaissance Orbiter, in Sixth International Conference on Mars, Abstract 3062, Lunar and Planet. Inst., Houston, Tex.

Pieters, C. M., and T. Hiroi (2004), RELAB (Reflectance Experiment LABoratory): A NASA Multiuser spectroscopy facility, Proc. Lunar Planet. Sci. Conf. 35th, Abstract 1720.

Poulet, F., and S. Erard (2004), Nonlinear spectral mixing: Quantitative analysis of laboratory mineral mixtures, J. Geophys. Res., 109, E02009, doi:10.1029/2003JE002179.

Press, W. H., S. A. Teukolsky, W. T. Vetterling, and B. P. Flannery (1992), Numerical Recipes in FORTRAN: The Art of Scientific Computing, 2nd ed., 963 pp., Cambridge Univ. Press, New York.

Rieder, R., et al. (2004), Chemistry of rocks and soils at Meridiani Planum from the alpha particle x-ray spectrometer, Science, 306, 1746-1749.

Roush, T. L. (1994), Charon: More than water ice?, Icarus, 108, 243-254

Roush, T. L. (1996), Mid-infrared $\left(5.0-25 \mu \mathrm{m}, 2000-400 \mathrm{~cm}^{-1}\right)$ optical constants of hydrous carbonate, sulfate, and nitrate, J. Geophys. Res. 101(E1), 2215-2224.

Roush, T. L. (2003), Estimated optical constants of the Tagish Lake meteorite, Meteorit. Planet. Sci., 38, 419-426.

Roush, T. L. (2005), Near-infrared $(0.67-4.7 \mu \mathrm{m})$ optical constants estimated for montmorillonite, Icarus, 179, 259-264

Roush, T. L., J. B. Pollack, F. C. Witteborn, J. D. Bregman, and J. P. Simpson (1990), Ice and minerals on Callisto: A reassessment of the reflectance spectra, Icarus, 86, 355-382.

Roush, T. L., J. Pollack, and J. Orenberg (1991), Derivation of mid-infrared $(5-25 \mu \mathrm{m})$ optical constants of some silicates and palagonite, Icarus, 94 $191-208$.

Salisbury, J. W., L. S. Walter, N. Vergo, and D. M. D'Aria (1991), Infrared

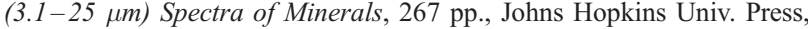
Baltimore, Md.

Shkuratov, Y. G., and Y. S. Grynko (2005), Light scattering by media composed of semitransparent particles of different shapes in ray optics approximation: Consequences for spectroscopy, photometry, and polarimetry of planetary regoliths, Icarus, 173, 16-28.

Shkuratov, Y., L. Starukhina, H. Hoffmann, and G. Arnold (1999), A model of spectral albedo of particulate surfaces: Implication to optical properties of the Moon, Icarus, 137, 235-246.

Squyres, S. W., et al. (2004), In situ evidence for an ancient aqueous environment at Meridiani Planum, Mars, Science, 306, 1709-1714.

Toon, O. B., J. B. Pollack, and B. Khare (1976), The optical constants of several atmospheric aerosol species: Ammonium sulfate, aluminum oxide, and sodium chloride, J. Geophys. Res., 81, 5733-5748.

Warren, S. G. (1984), Optical constants of ice from the ultraviolet to the microwave, Appl. Opt., 23, 1206-1225.

L. Colangeli and F. Esposito, INAF, Osservatorio Astronomico di Capodimonte, Via Moiariello 16, I-80131 Napoli, Italy.

G. R. Rossman, Division of Geological and Planetary Sciences, California Institute of Technology, Mail Stop 150-21,1200 E. California Blvd., Pasadena, CA 91125, USA.

T. L. Roush, Planetary Systems Branch, Space Sciences and Astrobiology Division, NASA Ames Research Center, MS 245-3, Moffett Field, CA 94035-1000, USA. (troush@mail.arc.nasa.gov) 